\title{
Density functional theory study of Fe(II) adsorption and oxidation on goethite surfaces
}

\author{
Benedict Russell, ${ }^{1, *}$ Mike Payne, ${ }^{1}$ and Lucio Colombi Ciacchi ${ }^{2,3,+}$ \\ ${ }^{1}$ Theory of Condensed Matter Group, \\ Cavendish Laboratory, University of Cambridge, \\ J J Thomson Avenue, Cambridge CB3 OHE, UK \\ ${ }^{2}$ Fraunhofer Institut für Werkstoffmechanik, \\ Wöhlerstrasse 11, 79108 Freiburg, Germany \\ ${ }^{3}$ Institut für Zuverlässigkeit von Bauteilen und Systemen, \\ Universität Karlsruhe, Kaiserstr. 12, 76131 Karlsruhe, Germany
}

(Dated: August 17, 2018)

\begin{abstract}
We study the interactions between Fe(II) aqua-complexes and surfaces of goethite $(\alpha$-FeOOH) by means of density-functional theory calculations including the so-called Hubbard $U$ correction to the exchange-correlation functional. Using a thermodynamic approach, we find that (110) and (021) surfaces in contact with aqueous solutions are almost equally stable, despite the evident needle-like shape of goethite crystals indicating substantially different reactivity of the two faces. We thus suggest that crystal anisotropy may result from different growth rates due to virtually barrier-less adsorption of hydrated ions on the (021) but not on the (110) surface. No clear evidence is found for spontaneous electron transfer from an adsorbed Fe(II) hex-aqua complex to a defect-free goethite substrate. Crystal defects are thus inferred to play an important role in assisting such electron transfer processes observed in a recent experimental study. Finally, goethite surfaces are observed to enhance the partial oxidation of adsorbed aqueous Fe(II) upon reaction with molecular oxygen. We propose that this catalytic oxidation effect arises from donation of electronic charge from the bulk oxide to the oxidizing agent through shared hydroxyl ligands anchoring the Fe(II) complexes on the surface.

PACS numbers: 71.15.Mb
\end{abstract}

Keywords: goethite surfaces, density functional theory, iron complexes, adsorption, oxidation 


\section{INTRODUCTION}

Goethite $(\alpha-\mathrm{FeOOH})$ is the most common iron (III) oxyhydroxide, and the only stable phase with respect to hematite and liquid water at ambient temperature and pressure conditions. $\frac{1}{-}$ It occurs naturally in soils as a result of weathering of other iron-based minerals, and is the dominant oxyhydroxide phase in lake and marine sediments. $\stackrel{2}{-}$ Due to its tendency to form nanoscale crystals with high specific surface area, goethite plays an important role in nature in controlling the mobility of heavy metals such as As, Cd, Zn, $\mathrm{Hg}$, as well as phosphate anions, and has been studied extensively as a model adsorbent in environmental science and technology $\frac{3,4,5}{2}$ It has also found applications as a precursor in the development of magnetic recording systems, being converted into maghemite $\left(\gamma-\mathrm{Fe}_{2} \mathrm{O}_{3}\right)^{\frac{6}{6}}$ or metallic particles ${ }^{7}$ by thermal treatment. Recently, it has been studied as a model colloidal system showing magnetically-sensitive liquid crystal behaviour $\underline{8}$

In technological applications, it is important both to have a control over the growth of nanoscale particles (e.g., to produce them with narrow size distributions and uniform properties), and to be able to predict the interactions between the particle surfaces and their external environment (e.g., to optimize their adsorption capabilities). However, the microscopic mechanisms responsible for the growth of goethite crystals from aqueous solutions are still poorly understood, $\stackrel{9}{,}$ and detailed electronic structure knowledge of iron oxyhydroxide surfaces is limited. ${ }^{10}$ This is partly due to the fact that quantum-mechanical calculations of iron oxyhydroxide phases are challenging for standard methods such as density functional theory (DFT) $, 11,12$ due to the complex magnetic structure and the large crystal unit cells which these phases present. Moreover, especially in the case of iron oxides, the strong electronic correlations arising from localised $d$-orbitals are not well described within the standard local-density (LDA) or generalized gradient (GGA) DFT approximations. $\underline{13}, \underline{14}, \underline{15}$ In the present paper we undertake an extensive study of the surface chemistry of goethite within the so-called LDA $+U$ scheme $16,17,18$, which integrates a model-Hamiltonian-based treatment of the localised $\mathrm{Fe}(3 d)$ orbitals within the framework of a GGA-DFT calculation. Our aim is to elucidate fundamental features of the mechanisms of goethite surface reactivity, in the context of crystal growth upon interaction with dissolved iron complexes.

Synthetic goethite may be produced either by precipitation from a saturated aqueous $\mathrm{Fe}(\mathrm{III})$ solution or by slow oxidation of aqueous $\mathrm{Fe}(\mathrm{II}) \stackrel{2}{2}$ It is well established that the 
(a)

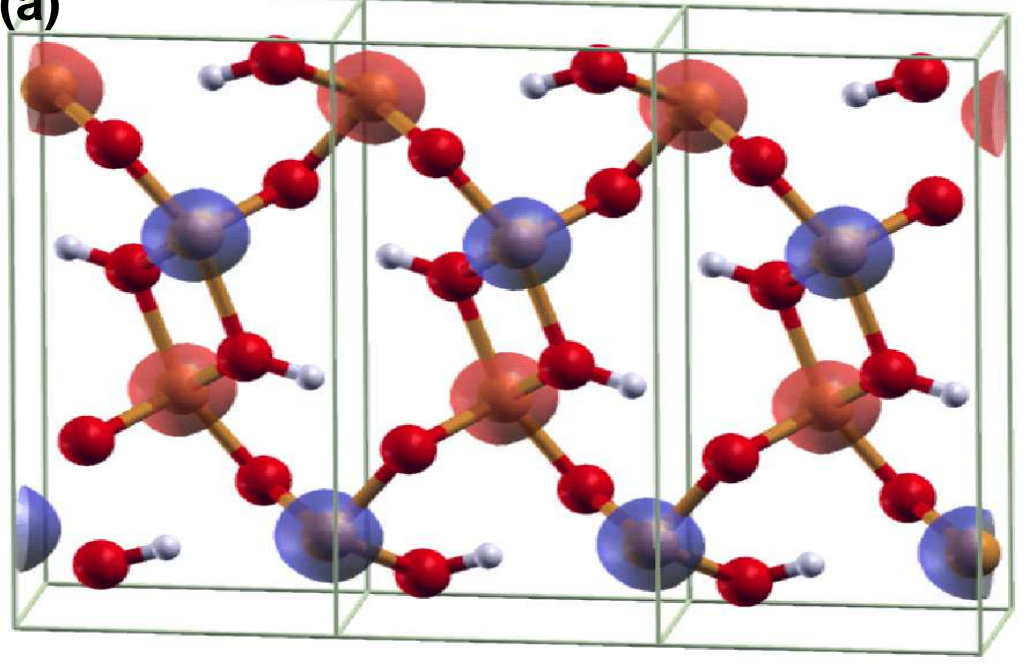

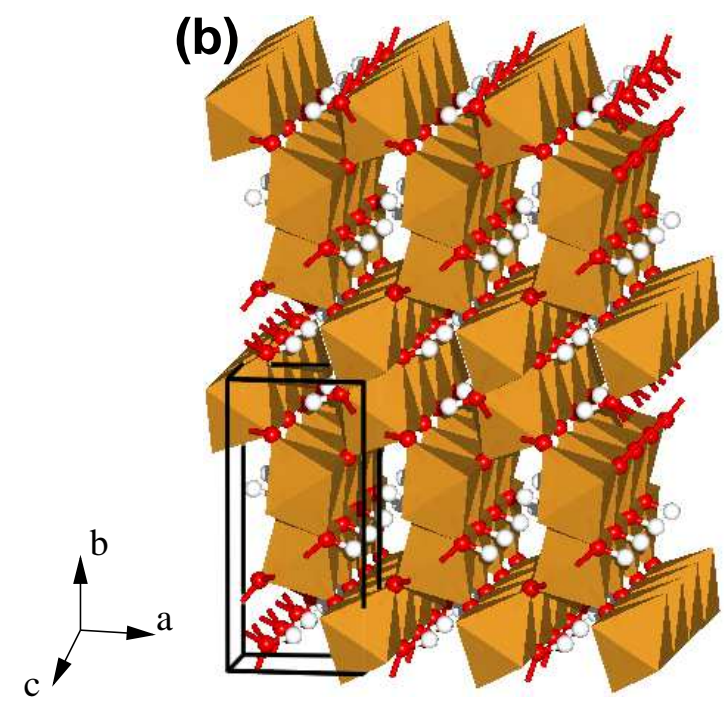

FIG. 1: (Color online) Two views of the structure of bulk goethite, showing the unit cell: (a) with spin density isosurface to show the antiferromagnetic arrangement of local spin moments in the ground state; (Red and blue indicate up and down spin, respectively.) (b) a polyhedral representation, illustrating the double chain structure of octahedrally coordinated Fe ions. Colors (online only): Fe gold, O red, $\mathrm{H}$ white.

oxidation of $\mathrm{Fe}(\mathrm{II})$ to $\mathrm{Fe}(\mathrm{III})$ in aqueous solution may be self-catalysed by already present iron oxyhydroxide particles, resulting in the growth of an $\mathrm{Fe}(\mathrm{III})$ layer on the oxide surface that is similar in structure to the underlying bulk material.9.10 However, the mechanisms of the surface redox reactions remain elusive. Recently, Williams and Scherer ${ }^{10}$ used Mössbauer spectroscopy to study the reaction of aqueous Fe(II) with Fe(III) oxide surfaces. Their study gives evidence of spontaneous electron transfer from the adsorbed Fe(II) complex to the underlying oxide, most probably occurring via overlap of $\mathrm{Fe}(3 d)$ orbitals in octahedral edgesharing environments on the crystal surface. However, whether this is an essential step in the catalytic oxidation of $\mathrm{Fe}(\mathrm{II})$ at the oxide surface, or whether it is in fact a competing process, was left as an unanswered question. Here we attempt to address this issue by performing electronic structure calculations of Fe(II) complexes adsorbing on goethite surfaces with different crystallographic orientations.

The crystal structure of goethite has been studied extensively by X-ray and neutron diffraction, and is shown in fig. 1. Goethite crystallises with an orthorhombic unit cell, with symmetry usually described by the space group Pbnm, $\stackrel{19}{ }$ though the orthorhombic group 

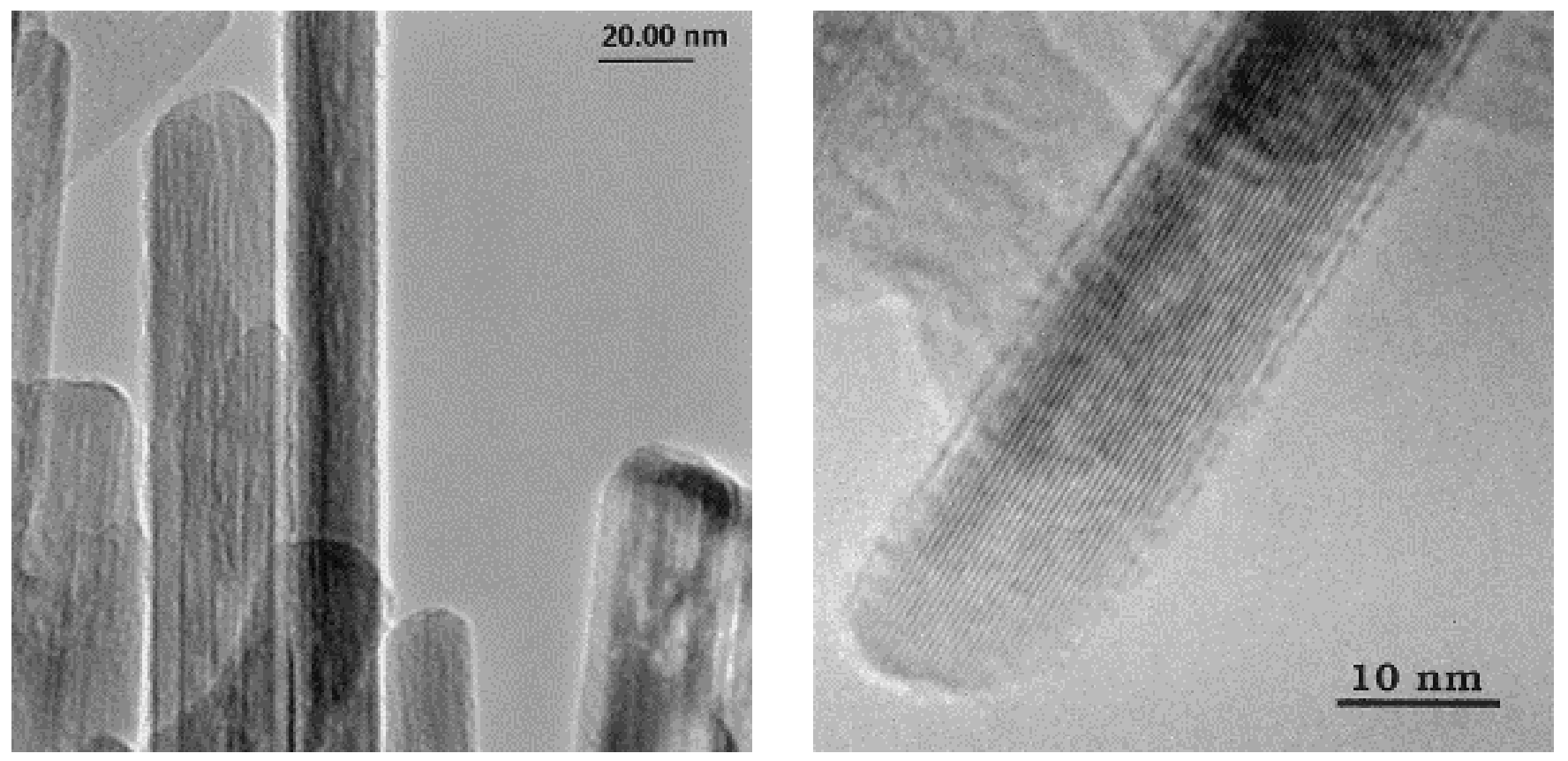

FIG. 2: TEM images of synthetic goethite crystals. Reprinted with permission from Elsevier $\stackrel{47}{\underline{47}}$

Pnma may be used equivalently: $\underline{20}$ The unit cell contains four FeOOH formula units, with ionic positions given by $\pm\left(x, y, \frac{1}{4}\right)$ and $\pm\left(\frac{1}{2}+x, \frac{1}{2}-y, \frac{3}{4}\right)$. The structure of goethite may be described as a slightly distorted hexagonally close-packed arrangement of oxygen and hydroxyl anions along the cell [100] axis, with Fe cations occupying half of the octahedral interstitial sites. In a polyhedral representation, it consists of parallel double chains of edgelinked $\mathrm{FeO}_{3}(\mathrm{OH})_{3}$ octahedra along the [001] direction, with neighboring chains linked to each other by corner-sharing. Below the Néel temperature of approximately 400K, goethite is antiferromagnetic, with local magnetic moments on the Fe ions alternating along the cell $b$-axis, and with the moments aligned parallel to the cell $c$-axis. $\underline{19}, 20$ Goethite is generally considered to be a charge-transfer insulator with a band gap of about $2.5 \mathrm{eV}$, the top of the valence band being dominated by $\mathrm{O}(2 p)$ states and the bottom of the conduction band having predominantly $\mathrm{Fe}(3 d)$ character. ${ }^{21}$

Natural and synthetic goethite crystals present a needle-like morphology, as illustrated by the transmission electron microscopy images in fig. 2. The crystal surface is usually made up mostly of (110) faces running parallel to the long axis of the needles, while the ends of the needles are capped predominantly by (021) and other planes with a large component perpendicular to the cell $c$-axis..$^{22,23,24,25,26}$ The $\{110\}$ and $\{021\}$ families might therefore 
represent surfaces with distinctly different character: the former being relatively stable and the latter providing a site for active crystal growth. In the remainder of the paper, we will focus on these two surfaces. In section [II we will study their structure and relative stability. In section IV we will then look for evidence of spontaneous oxidation of Fe(II) hexaqua complexes adsorbing on them. Finally, in section $\nabla$ we will investigate the possibility of surface-catalyzed oxidation via reaction of the adsorbed complexes with molecular oxygen.

\section{METHODS}

\section{A. Density Functional Calculations}

The calculations described in this paper have been performed using the CASTEP simulation package,$\frac{27}{2}$ which provides an implementation of spin-polarized DFT based on the plane-wave pseudopotential scheme..$^{28}$ Exchange and correlation were treated within the generalized gradient approximation (GGA), using the functional form of Perdew, Burke and Enzerhof (PBE) ${ }^{29}$ Ultrasoft pseudopotentials $\stackrel{30,31}{ }$ were used to describe the ionic cores $\left(1 s^{2}\right.$ for $\mathrm{O}, 1 s^{2} 2 s^{2} 2 p^{6} 3 s^{2} 3 p^{6}$ for $\mathrm{Fe}$ ), and non-linear core corrections ${ }^{32}$ were applied for iron to improve the description of magnetically polarized states. The electronic Kohn-Sham wave functions were expanded using a plane-wave basis set, up to a kinetic energy cut-off of $450 \mathrm{eV}$, which was shown to converge the formation energy of bulk goethite to within $1 \mathrm{meV}$ per atom with respect to increasing basis size. Monkhorst-Pack grids ${ }^{33}$ were used to sample the Brillouin zone. In the case of bulk goethite, a $4 \times 2 \times 6$ grid was used, giving convergence of better than $0.1 \mathrm{meV}$ in the total energy per formula unit.

The LDA $+U$ formalism has been implemented within CASTEP according to the scheme described by Cococcioni et al. $\stackrel{34}{35}$ Within this scheme, the Hubbard $U$ is not treated as an empirical fitting parameter, but may rather be determined self-consistently from the calculated ground state. In principle, the value of $U$ should be determined separately for each system studied. However, it would be inappropriate to make direct comparisons between total energies from calculations using differing values of $U$. For this reason, we have instead determined the self consistent value for Fe in bulk goethite, and then used this same value for the subsequent surface calculations. The possible impact of using a different value for the $U$ parameter is considered briefly in section IVB. 


\section{B. Thermodynamic Approach}

By means of DFT calculations, we can readily obtain total energies at zero temperature for a range of surface configurations. However, in order to make a more meaningful comparison of the relative stability at finite temperature of surface structures of different stoichiometry, we take a thermodynamical approach, which we outline below. A more detailed discussion relating to the integration of thermodynamics with electronic structure calculations may be found, for example, in Ref. 36.

Conceptually, we consider a system consisting of three regions: a large region of bulk goethite with stoichiometry $\mathrm{FeOOH}$, a large reservoir of liquid water at neutral $\mathrm{pH}$ and a surface region of variable stoichiometry. The most stable surface configuration at given temperature, $T$, and pressure, $p$, is that which minimizes the surface free energy, $\gamma(T, p)$, given, in the case of a slab model with two equivalent surfaces, by

$$
\gamma(T, p)=\frac{1}{2 A}\left[G_{\text {slab }}\left(T, p, N_{\mathrm{Fe}}, N_{\mathrm{O}}, N_{\mathrm{H}}\right)-N_{\mathrm{Fe}} \mu_{\mathrm{Fe}}(T, p)-N_{\mathrm{O}} \mu_{\mathrm{O}}(T, p)-N_{\mathrm{H}} \mu_{\mathrm{H}}(T, p)\right],
$$

where $\mu_{\mathrm{Fe}}, \mu_{\mathrm{O}}, \mu_{\mathrm{H}}$ are the chemical potentials for Fe, $\mathrm{O}$ and $\mathrm{H}$ atoms, respectively, $N_{\mathrm{Fe}}, N_{\mathrm{O}}$, $N_{\mathrm{H}}$ are the numbers of atoms of each species making up the surface region, and $A$ is the area of the surface unit cell.

At chemical equilibrium, we can impose relationships between the chemical potentials of $\mathrm{Fe}, \mathrm{O}$ and $\mathrm{H}$ :

$$
\begin{aligned}
\mu_{\mathrm{Fe}}+2 \mu_{\mathrm{O}}+\mu_{\mathrm{H}} & =g_{\mathrm{FeOOH}}^{\text {bulk }}, \\
2 \mu_{\mathrm{H}}+\mu_{\mathrm{O}} & =g_{\mathrm{H}_{2} \mathrm{O}}^{\text {liquid }},
\end{aligned}
$$

where $g$ represents a Gibbs free energy per formula unit. All the surface configurations considered in this paper may be constructed stoichiometrically from $\mathrm{FeOOH}$ and $\mathrm{H}_{2} \mathrm{O}$, and hence we can rewrite eq. (1) as:

$$
\gamma(T, p)=\frac{1}{2 A}\left[G_{\text {slab }}\left(T, p, N_{\mathrm{FeOOH}}, N_{\mathrm{H}_{2} \mathrm{O}}\right)-N_{\mathrm{FeOOH}} g_{\mathrm{FeOOH}}^{\text {bulk }}(T, p)-N_{\mathrm{H}_{2} \mathrm{O}} g_{\mathrm{H}_{2} \mathrm{O}}^{\text {liquid }}(T, p)\right] .
$$

The Gibbs free energies, $G_{\text {slab }}, g_{\mathrm{FeOOH}}^{\text {bulk }}$ and $g_{\mathrm{H}_{2} \mathrm{O}}^{\text {liquid }}$, may be related to the total energy, $E^{e l}$, obtained in a typical DFT calculation, by

$$
G=E^{e l}+E^{v i b}+p V-T S
$$


For pressures of the order of 1 atm, and surface relaxations of the order of $1 \AA$, the contribution from the $p V$ term to $\gamma$ is of the order of $0.001 \mathrm{meV} / \AA^{2}$, and may therefore safely be neglected. For solid materials, the remaining terms, $E^{v i b}-T S$, arise principally from lattice vibrations (phonons). In this work, we assume that the phonon density of states of the solid is not significantly altered by the surface configuration, so that contributions from the slab and from the bulk will cancel out to a large extent in determining the surface free energy. Hence, for the purpose of comparing surface free energies of different faces, we take the DFT total energies of the slab and the bulk as a direct approximation to the corresponding Gibbs free energies.

In order to estimate $g_{\mathrm{H}_{2} \mathrm{O}}^{\text {liquid }}(T, p)$, we combine a well converged DFT total energy for a single water molecule, $E_{\mathrm{H}_{2} \mathrm{O}}^{e l}$, with an experimental Gibbs free energy of solvation, $\Delta g_{\mathrm{H}_{2} \mathrm{O}}^{\text {solv }}$, so that:

$$
g_{\mathrm{H}_{2} \mathrm{O}}^{\text {liquid }}(T, p)=E_{\mathrm{H}_{2} \mathrm{O}}^{e l}+\Delta g_{\mathrm{H}_{2} \mathrm{O}}^{\text {solv }}(T, p) .
$$

In an aqueous environment, we should also consider the free energy of solvation of the surface, $\Delta G_{s l a b}^{\text {solv }}$. However, the chosen surface terminations represent in some sense an explicit consideration of the first stages of hydration of the bare surfaces, and the fully hydroxylated (110) and (021) surfaces have a very similar density of surface anion groups (approximately $15 \mathrm{~nm}^{-2}$ in both cases). Thus, even if the solvation free energy is a significant fraction of the overall surface energy, it is unlikely to contribute significantly to the relative stability of these surfaces. For this reason, no attempt has been made to include $\Delta G_{\text {slab }}^{\text {solv }}$ in the results presented in this paper.

We thus arrive at our final expression:

$$
\gamma(T, p) \simeq \frac{1}{2 A}\left[E_{\text {slab }}^{e l}-N_{\mathrm{FeOOH}} E_{\mathrm{FeOOH}}^{e l}-N_{\mathrm{H}_{2} \mathrm{O}}\left(E_{\mathrm{H}_{2} \mathrm{O}}^{e l}+\Delta g_{\mathrm{H}_{2} \mathrm{O}}^{\text {solv }}(T, p)\right)\right]
$$

In the results that follow, we use a value $\Delta g_{\mathrm{H}_{2} \mathrm{O}}^{\text {solv }}(298 \mathrm{~K}, 1 \mathrm{~atm})=-0.274 \mathrm{eV} /$ molecule, taken from Ref. 37. The value of $E_{\mathrm{FeOOH}}^{e l}$ is taken from the calculations described in section IIIA, while $E_{\mathrm{H}_{2} \mathrm{O}}^{e l}$ is obtained from a geometry optimization of an isolated water molecule in a $16 \AA$ cubic supercell. In both cases, the same cut-off energy, pseudopotentials and exchange-correlation functional were used as for the main calculations. 
TABLE I: Calculated and average experimental cell parameters, magnetic moments and relative energy per formula unit for different magnetic structures of goethite.

\begin{tabular}{ll|lll|l}
\hline \hline & & $a(\AA)$ & $b(\AA)$ & $c(\AA)$ & $\Delta E(\mathrm{meV})$ \\
\hline Exp. & $\mathrm{AF}$ & 4.625 & 9.963 & 3.023 & \\
\hline GGA & $\mathrm{AF}$ & 4.660 & 9.987 & 3.006 & 0 \\
& $\mathrm{FM}$ & 4.402 & 9.594 & 2.898 & +100 \\
& $\mathrm{NM}$ & 4.369 & 9.514 & 2.908 & +155 \\
\hline $\mathrm{GGA}+U$ & $\mathrm{AF}$ & 4.646 & 10.150 & 3.084 & \\
\hline \hline
\end{tabular}

\section{GOETHITE SURFACES}

In this section we will present results on the thermodynamic stability of goethite surfaces in equilibrium with water solutions. After a brief description of the bulk properties of goethite crystals, structural and energetic details of surfaces will be described and discussed in the context of crystal growth from dissolved iron ions.

\section{A. Bulk Goethite}

In our GGA-DFT calculations, we have taken into account five possible magnetic phases of goethite: a non-magnetic (NM) phase, a ferromagnetic (FM) phase and three antiferromagnetic (AFM) phases differing in the ordering of up and down local spin moments within the cell. The cell parameters and energies resulting from structural optimizations of each phase are shown in Table I.

In agreement with experimental results, $\frac{19,20}{2}$ we find an AFM ground state with local spin moments of the Fe ions alternating along the cell $b$-axis. The optimized cell parameters and atomic coordinates agree to within $1 \%$ and $0.3 \%$, respectively, with the experimental values (Tables 【 and 【I). By varying the cell volume around the equilibrium value and fitting a Murnaghan equation of state to the resulting total energies, the bulk modulus is estimated to be $89 \mathrm{GPa}$. As is often found in GGA calculations, this is considerably lower than the recently reported experimental value of $111 \mathrm{GPa} . \underline{38}$

For the minimum energy structure, the electronic density of states (DOS) projected onto 
TABLE II: Calculated and experimental ionic positions for antiferromagnetic goethite. Experimental values from Ref. 19

\begin{tabular}{l|ll|ll|ll}
\hline \hline \multirow{2}{*}{ Species } & \multicolumn{2}{|l}{ Experimental } & \multicolumn{2}{c}{ GGA } & \multicolumn{2}{c}{ GGA $+U$} \\
\cline { 2 - 6 } & $y$ & $x$ & $y$ & $x$ & $y$ \\
\hline $\mathrm{Fe}$ & 0.0477 & 0.8539 & 0.0465 & 0.8509 & 0.0633 & 0.8559 \\
$\mathrm{O}_{I}$ & 0.7058 & 0.1994 & 0.7039 & 0.2015 & 0.6775 & 0.1937 \\
$\mathrm{O}_{I I}$ & 0.1974 & 0.0531 & 0.1965 & 0.0529 & 0.1871 & 0.0585 \\
$\mathrm{H}$ & 0.3991 & 0.0876 & 0.3982 & 0.0847 & 0.3899 & 0.0947 \\
\hline \hline
\end{tabular}

$\mathrm{Fe}(3 d)$ and $\mathrm{O}(2 p)$ atomic orbitals (Fig. 5) reveals the presence of a band gap of $0.8 \mathrm{eV}$, which is significantly smaller than the experimental value of $2.5 \mathrm{eV}$. We also observe strong mixing of the majority-spin $\mathrm{Fe}(3 d)$ states with the $\mathrm{O}(2 p)$ states across the whole range of the valence band, in conflict with the experimental observation that the valence band edge should be dominated by $\mathrm{O}(2 p)$ states. The predominantly $\mathrm{Fe}(3 d)$ nature of the conduction band is correctly reproduced. Hybridization of the atomic orbitals in the crystal environment results in a non-negligible contribution to the density of states below the Fermi level from minority spin $\mathrm{Fe}(3 d)$-like states. However, there is no sharp peak below the Fermi level corresponding to a single occupied minority spin $\mathrm{Fe}(3 d)$ orbital, as typically found in the case of of $\mathrm{Fe}^{2+}$ ions. This fact, together with the complete occupation of the majority spin $\mathrm{Fe}(3 d)$ orbitals, is consistent with the identification of goethite as an Fe(III) compound.

In order to improve the description of the electronic properties, we have performed additional calculations for the AFM ground state at the GGA $+U$ level. The value of the Hubbard $U$ parameter was determined self-consistently according to the procedure of Cococcioni et

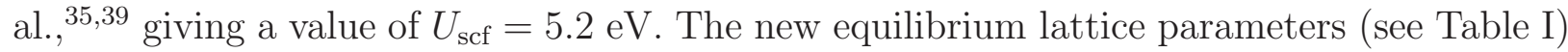
are on average slightly further from the most recent experimental results than the GGA parameters, but the agreement, within $1.5 \%$, is still reasonable. With the $U$ correction, the calculated bulk modulus is $109 \mathrm{GPa}$, very close to the experimental value. The optimized ionic positions (see Table II) present a maximum deviation of $3 \%$ (average 1\%) from the experimental values. We note that our self-consistent value of $U$ is implicitly chosen to correct the electronic properties rather than the structural properties, which were in any 
case well described at the GGA level. Indeed, as reported previously by other authors, it is often not possible to choose a single value of $U$ that gives quantitatively correct predictions of both the structural and electronic properties. $\stackrel{40}{ }$

With this in mind, we have calculated the GGA $+U$ electronic DOS using the relaxed structure obtained from the GGA calculations. The results, projected as before onto Fe(3d) and $\mathrm{O}(2 p)$ orbitals, are shown in fig. 5b. The addition of the on-site repulsion term to the Kohn-Sham Hamiltonian has a strong influence on the electronic structure around the Fermi level compared with the GGA results. The unoccupied Fe(3d) minority spin states are pushed to higher energies, thus increasing the band gap from the GGA value of $0.8 \mathrm{eV}$ to $2.5 \mathrm{eV}$. At the same time, the occupied $\mathrm{Fe}(3 d)$ majority spin states move further below the Fermi level, breaking the strong $\mathrm{Fe}(3 d)-\mathrm{O}(2 p)$ hybridization observed in the GGA DOS. Thus, GGA $+U$ predicts for Goethite, in full agreement with experiment, a charge transfer insulating state with a band gap of $2.5 \mathrm{eV}$ between $\mathrm{O}(2 p)$-dominated states at the top of the valence band and localized $\mathrm{Fe}(3 d)$ minority spin states at the bottom of the conduction band.

The GGA $+U$ DOS also shows a reduction in the partial occupation of minority spin $\mathrm{Fe}(3 d)$ states relative to the GGA electronic structure, making the identification of the Fe(III) oxidation state even clearer than before. This also leads to an increase in the local magnetic moment on the Fe ions from $3.74 \mu_{B}$ to $4.16 \mu_{B}$. (To the best of our knowledge, no experimental information on the value of the magnetic moments is available for wellcrystallized goethite, although Bocquet and Kennedy found a saturation magnetic moment of $3.80 \mu_{B}$ per iron atom for fine particle goethite. $\left.\stackrel{41}{ }\right)$

\section{B. Goethite surfaces}

\section{Surface terminations}

Previous theoretical studies of goethite surfaces have assumed complete hydroxylation of the surface. $\stackrel{42}{*}$ Fully hydroxylated surfaces can be considered as arising from a truncation of the bulk such that all Fe ions remain octahedrally coordinated, followed by the addition of sufficient protons to cancel the excess negative charge of the surface layer. In this study, we take a slightly different approach which allows us also to investigate a range of intermediate 
stages of hydroxylation. Starting from stoichiometric, non-polar terminations of the (110) and (021) surfaces, we consider the results of heterolytic dissociation and adsorption of water molecules, obtaining a total of five different surface configurations for the two surfaces, as outlined below. Here we implicitly assumed a solution at neutral pH, so that only whole water molecules (undissociated or dissociated) are used to terminate the surfaces.

For the (110) surface, a stoichiometric truncation of the bulk (fig. 3a) leads to two inequivalent Fe surface sites, one five-fold coordinated and the other six-fold coordinated. Adjacent six-fold coordinated sites are joined by bridging oxygen atoms. The dissociative addition of a single water molecule is then sufficient to fully hydroxylate the surface, with the hydroxyl group binding to the formerly under-coordinated Fe and the proton donated to the bridging oxygen atom (fig. 3b). The bare (021) surface (fig. 3r) also presents two inequivalent Fe sites, in this case both five-fold coordinated. One (A) is bound to 3 oxygens and 2 hydroxyl groups, while the other (B) is bound to 2 oxygens and 3 hydroxyl groups. The surface anion layer takes the form of bridging oxygen atoms and hydroxyl groups. We consider two steps of hydroxylation: first, the dissociative addition of a single water molecule per two Fe sites, with the hydroxyl group binding to the B site and the proton donated to the bridging oxygen atom (fig. $3 \mathrm{~d}$ ), and second, the adsorption of an additional water molecule onto the Fe A site (fig. 3 $)$.

\section{Surface structure}

Starting from the GGA-relaxed goethite bulk structure, the geometries of all five surfaces were optimized at the GGA level. Using the optimized geometries, single point electronic minimizations were also performed at the $\mathrm{GGA}+U$ level, using the same value of $U=5.2 \mathrm{eV}$ for the $\mathrm{Fe}(3 d)$ orbitals as for the goethite bulk. In all cases, a slab model was employed, with

neighboring slabs separated from each other by a vacuum region of at least $5.5 \AA$. Doubling the separation between the slabs was shown to change the resulting surface energies by less than $1 \mathrm{meV} / \AA^{2}$. For both the (110) and the (021) surfaces, the slab contained four layers of Fe ions, while the cell vectors in the plane of the surface corresponded to a single unit cell of the bulk structure. Brillouin zone sampling was performed by means of Monkhorst-Pack grids in the plane of the surface, using $8 \times 2$ points for the $(110)$ surfaces and $4 \times 2$ for the (021) surfaces. The relaxed structures for the three (021) and the two (110) surfaces are 
(a)

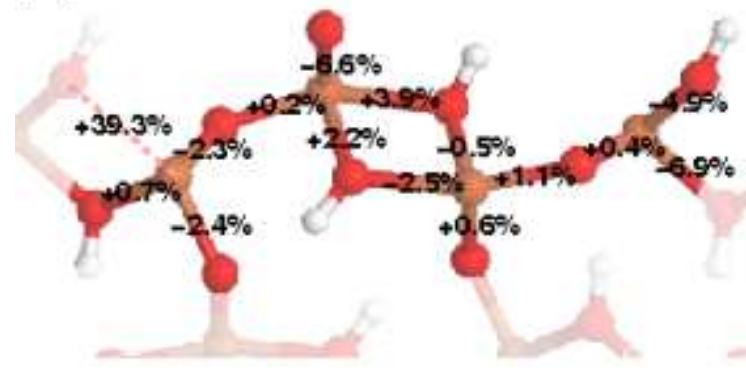

(c)

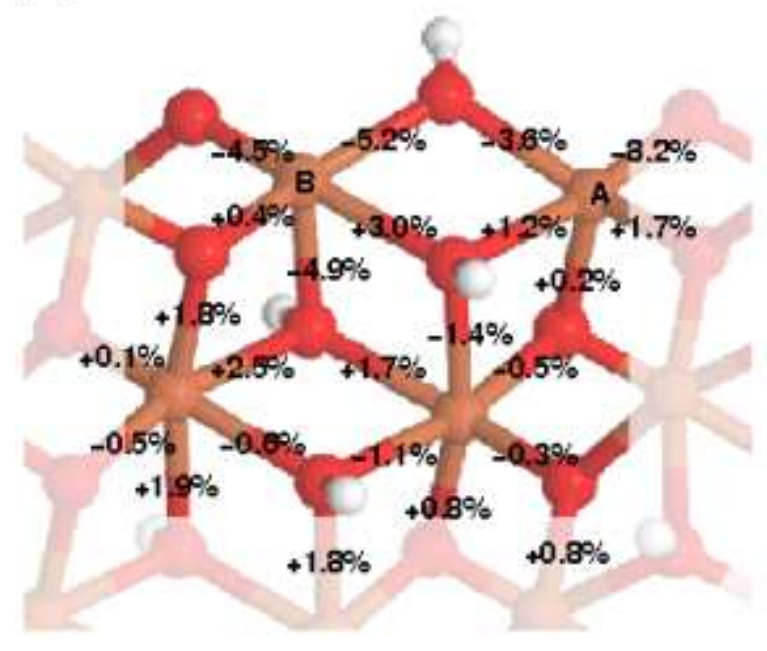

(b)

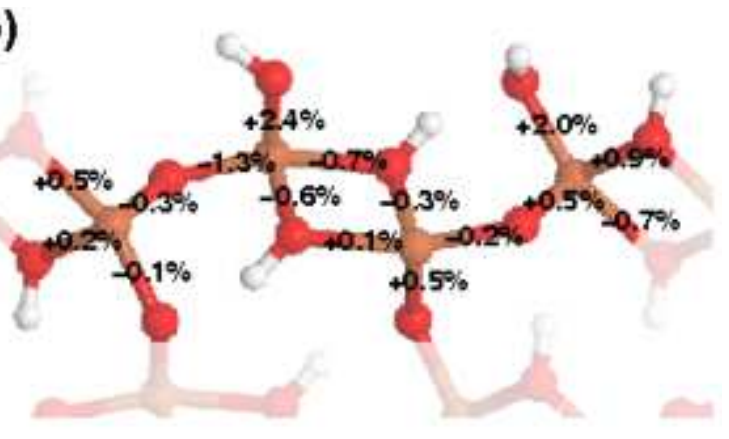

(d)

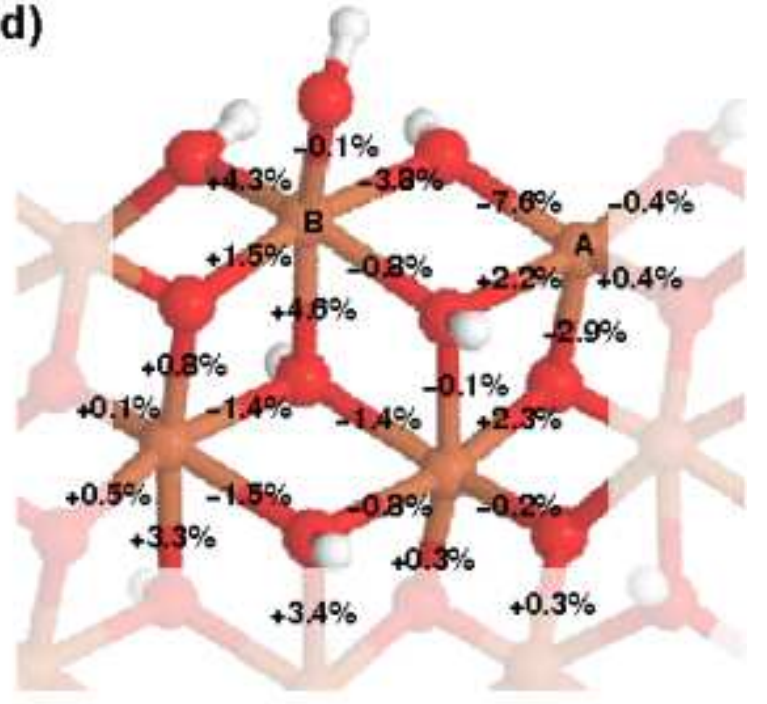

(e)

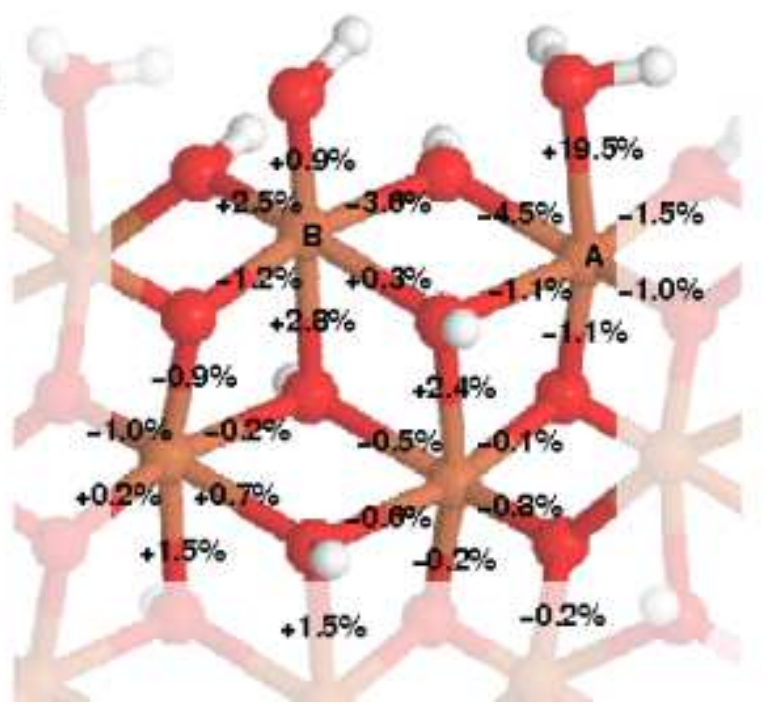

FIG. 3: (Color online) Changes in $\mathrm{Fe}-\mathrm{O}$ bond lengths relative to corresponding values in the bulk for the (a) bare and (b) hydroxylated (110) surfaces and (c) bare, (d) partially hydroxylated and (e) fully hydroxylated (021) surfaces of goethite, as determined by GGA geometry optimizations. 
shown in Fig. 3 along with the differences in bond lengths with respect to bulk goethite.

For the bare (021) surface, the under-coordination results in a reduction of the Fe-O distances. In the bare (110) surface, beside a reduction of the Fe-O distances, more drastic relaxations are observed. The under-coordinated Fe ions at the surface are pulled in towards the bulk. In the subsequent rearrangement, formerly three-fold coordinated hydroxyl groups break one of their Fe-O bonds to adopt a bridging configuration between neighboring fivefold coordinated Fe ions.

In the partially hydroxylated (021) surface, the protonation of the bridging oxide groups causes an increase in the corresponding $\mathrm{Fe}-\mathrm{O}$ bond lengths, while the bridging $\mathrm{OH}$ groups are displaced from their symmetrical positions, with new bond lengths of 2.03 and $1.97 \AA$ to the six-fold and five-fold coordinated Fe ions, respectively. The newly added terminal $\mathrm{OH}$ group has a bond length of $1.97 \AA$ to the surface. In the fully hydroxylated (021) surface, the additional water ligands are only weakly bound to the surface, with a Fe-O distance of $2.53 \AA$. However, this is sufficient to satisfy the under-coordination of the surface Fe ions. The bridging anions thus return to symmetrical positions, while the terminal OH group moves away slightly from the surface, with a new Fe-O bond length of $1.99 \AA$. As expected, the hydroxylated (110) surface shows the smallest relaxations among all five surfaces, with only the outermost anion layer showing bond length changes greater than $1 \%$ relative to the bulk. This justifies the use of a relatively thin slab for the study of this surface.

\section{Surface stability and crystal growth}

The surface Gibbs free energies of each of the five surfaces, calculated relative to bulk goethite and liquid water as described in Section [IB, are reported in Table III. It is clear that the hydroxylated surfaces are in general strongly favored over the bare surfaces both at the GGA and at the GGA+U levels, with surface energy values between 10 and $20 \mathrm{meV} / \AA^{2}$. These are relatively small compared with typical values calculated for other iron oxide phases. For example, for all trivial surface terminations of the hydroxylated hematite(0001) surface Trainor et al. computed surface free energies greater than $50 \mathrm{meV} / \AA^{2}$ at low oxygen partial pressure $\stackrel{43}{*}$ For the same surface in a dry environment, Wang et al. computed a surface energy above $90 \mathrm{meV} / \AA^{2}$ in all cases except in very oxygen-rich conditions, in which case a minimum surface energy of $45 \mathrm{meV} / \AA^{2}$ was observed for the oxygen-terminated surface. $\stackrel{44}{ }$ 
TABLE III: Calculated surface free energies, $\gamma$, for (110) and (021) surfaces of goethite with a number of different surface terminations. The geometry of all surfaces was optimized at the GGA level only. $U=5.2 \mathrm{eV}$ for GGA $+U$ calculations.

\begin{tabular}{l|lc}
\hline \hline & \multicolumn{2}{|c}{$\gamma\left(\mathrm{meV} / \AA^{2}\right)$} \\
& GGA GGA $+U$ \\
\hline (110) bare & 49 & 67 \\
(110) hydroxylated & 21 & 14 \\
\hline (021) bare & 34 & 46 \\
(021) partially hydroxylated & 20 & 14 \\
(021) fully hydroxylated & 16 & 12 \\
\hline \hline
\end{tabular}

Finally, similar differences in surface energy between the dry or hydroxylated surfaces of goethite and hematite have been recently calculated within the Born model of solids. $\underline{45}$ These results may be consistent with the observation that goethite is commonly formed as the first precipitate from solution even when hematite is the thermodynamically stable bulk phase. Indeed, in the initial nucleation stages a phase with very low surface energy could be favored over a phase with a surface energy too large to be compensated for by the energy gained from forming the bulk material.

It is intriguing, given the evident anisotropy of goethite crystals (see Fig. 2), that the (110) and (021) surfaces present roughly the same surface energy. Unless very different oxygen terminations that those considered here govern the behaviour of the experimentally investigated systems, our finding suggests that the needle-like crystal shape may result not from unequal thermodynamical surface stabilities but from unequal growth rates, with the (021) surface growing outwards faster than the (110) surface. $\frac{9}{}$ In an attempt to rationalize this hypothesis, we note that our calculated energies of the partially and fully hydroxylated (021) surfaces differ by less than $4 \mathrm{meV} / \AA^{2}$. This corresponds to a binding energy per added water molecule of just $-0.10 \mathrm{eV}$, less than half the energy of a typical single hydrogen bond in liquid water $\underline{\underline{46}}$ Therefore, the terminal water positions may be only partially occupied when averaged over time, giving the possibility of nearly barrier-less adsorption of additional $\mathrm{Fe}^{3+}$ or $\mathrm{Fe}^{2+}$ ions at these sites. In contrast, the corresponding sites on the (110) surface are 
occupied by stably bound hydroxyl ligands. Some form of ligand exchange would thus be required for an additional ion to bind to this surface, which would introduce an associated energy barrier.

Notably, goethite crystals take approximately the same form regardless of whether they are grown by precipitation from an $\mathrm{Fe}(\mathrm{III})$ solution $\underline{\underline{47}}$ or by oxidation of an Fe(II) solution $\underline{\underline{9}}^{-1}$ However, in the latter case another possible contribution to unequal growth rates would be preferential oxidation of Fe(II) to Fe(III) on (021) surfaces as compared with (110) surfaces. The oxidation of $\mathrm{Fe}(\mathrm{II})$ at these goethite surfaces is addressed in the remaining sections of this paper.

\section{SPONTANEOUS OXIDATION UPON ADSORPTION}

$\mathrm{Fe}(\mathrm{III})$ oxide surfaces are thought to promote the autocatalytic oxidation of Fe(II) ions during crystal growth.$\underline{9}$ In this section, we aim to investigate the adsorption of a Fe(II) complex on the (110) and (021) goethite surfaces, in particular looking for possible spontaneous electron transfer processes to the Fe(III) solid from the adsorbed ion.

Owing to the large computational cost associated with our first-principles approach, an exhaustive search for the lowest energy conformation of an Fe(II) ions adsorbing on each surface is not presently feasible. However, it has been observed experimentally that the binding of octahedral metal complexes on iron oxyhydroxide surfaces strongly favors adsorption

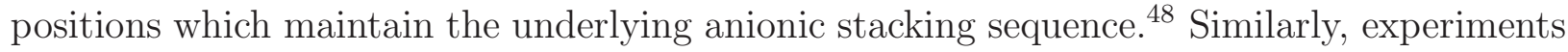
have shown that the oxide layer formed when aqueous Fe(II) ions are adsorbed and oxidized on a Fe(III) oxyhydroxide surface is generally similar in structure to the underlying bulk oxyhydroxide. ${ }^{10}$ As explained here below, applying these considerations allows us to consider only one adsorbed configuration on each of the two surfaces, which can both be considered to be fully hydroxylated in light of the surface energies computed in the previous section.

On the (110) surface, the complex may bind either through a single bridging hydroxyl group on the surface or through two terminal hydroxyl groups, corresponding to single or double corner sharing, respectively, in terms of the $\mathrm{Fe}\left(\mathrm{O}, \mathrm{OH}, \mathrm{H}_{2} \mathrm{O}\right)_{6}$ coordination octahedra. Both sites may be filled independently, but we may reasonably expect a stronger interaction between the complex and the surface in the double corner sharing case. The relaxed structure

for this site is shown in fig. 4 a. The (021) surface, as previously described, represents a 

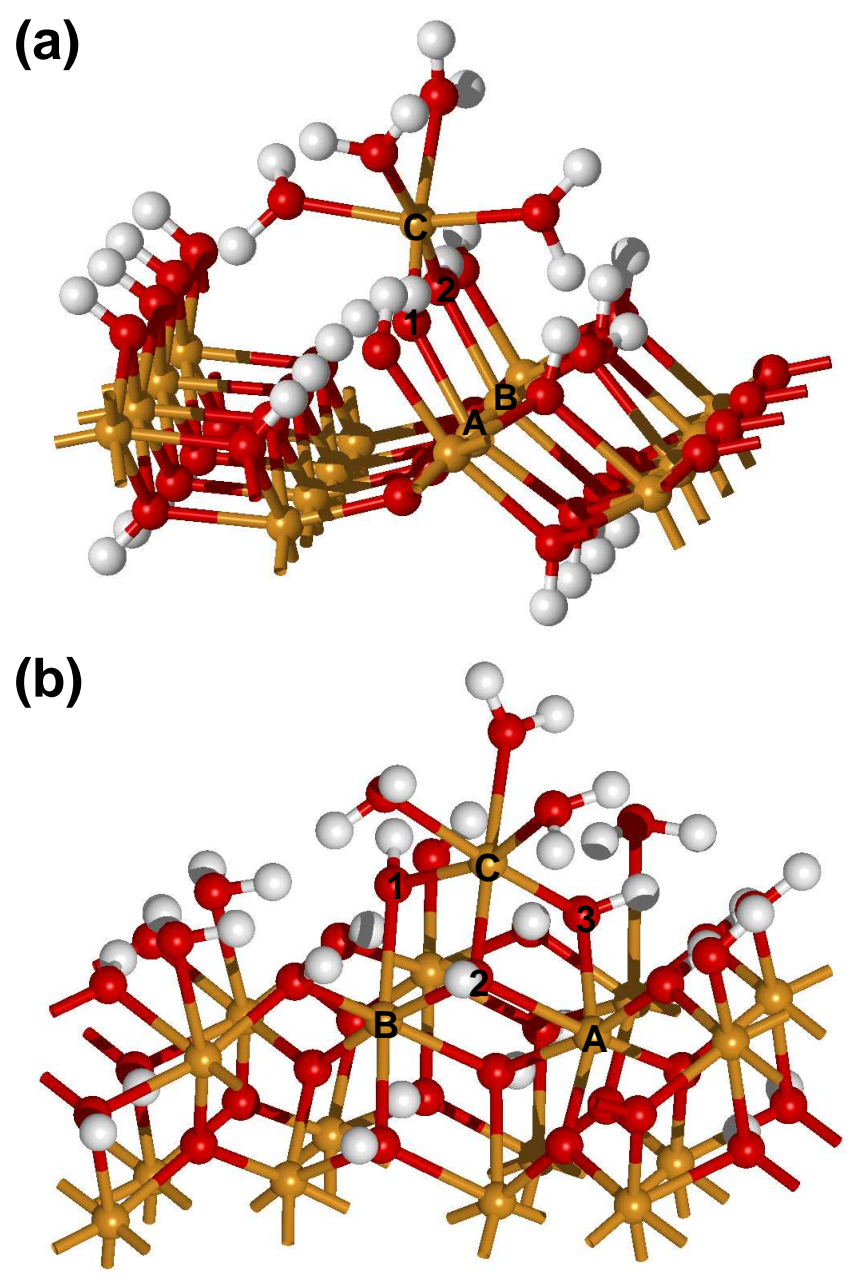

FIG. 4: (Color online) The GGA relaxed structure of $\left[\mathrm{Fe}\left(\mathrm{H}_{2} \mathrm{O}\right)_{6}\right]^{2+}$ bound to the goethite (110) surface in a double corner sharing configuration (a) and to the goethite (021) surface in a double edge sharing configuration (b).

termination of the double chains of octahedra composing the bulk. We thus consider the addition of a new octahedron to the end of a double chain, respecting the intrinsic staggered stacking sequence. This leads to the adsorbed ion configuration shown in fig. 4 $\mathrm{b}$, in which two octahedral edges (i.e. three anion groups) are shared between the complex and the surface. In order to reduce unfavorable steric clashes, a water ligand which occupied a bridging position between the surface and the complex is replaced with a hydroxyl group. While this allows the complex to adopt a less strained position on the surface, preliminary electronic structure calculations showed that this deprotonation does not influence the oxidation state of the adsorbed complex. 
Simulation cells were constructed by starting from the relaxed surface structures obtained in section IIIB and expanding the unit cell to enable the adsorption of a single complex without significant interaction between the complex and its periodic images in neighboring cells. For the (110) surface, the new cell consisted of four original surface cells, and had surface dimensions $11.0 \times 12.0 \AA^{2}$. For the $(021)$ surface, the original cell was doubled to give surface dimensions $9.4 \times 11.7 \AA^{2}$. In both cases, the number of k-points in the plane of the surface was reduced to a $2 \times 2$ grid. The vacuum region was also slightly widened to make room for the complex, ensuring a $5 \AA$ separation from the opposing surface in the adjacent cell. For both surfaces, we have assumed the Fe ion in the bound complex to be in a high spin state, since both the initial state (the unbound Fe(II) complex) and the final state (Fe(III) in bulk goethite) fall into this category. Thus the total starting spin of the system was set to $4 / 2$, equal to that of the isolated complex.

The structure of both systems was relaxed using GGA DFT calculations (fig. 4). Relaxed bond lengths compared with corresponding values in bulk goethite are given in table IV] Using the relaxed geometries, the electronic structure was studied at both the GGA and GGA $+U$ levels with $U=5.2 \mathrm{eV}$. Local atomic charges have been calculated according to the Bader partitioning scheme ${ }^{\frac{49}{4}}$ using a grid-based algorithm developed by Henkelman et al. ${ }^{50,51}$ In our calculations we favored the Bader scheme over the Mulliken partitioning scheme ${ }^{52}$ because we found it to be more resistant to the difference between molecular and crystalline environments. The Bader charges, coupled with information from the DOS projection of the $\mathrm{Fe}(3 d)$ orbitals, are indicative of the oxidation state of the complex upon adsorption on the surface. The results for the two systems are presented in the following two sections.

\section{A. Double corner sharing on the (110) surface}

After binding to the (110) surface, the Bader charge of the Fe ion in the complex shows a slight increase, from 1.61 to 1.68 e. The two neighboring surface Fe ions, with which the adsorbed complex shares two hydroxyl ligands, show a correspondent reduction in charge from 1.86 to 1.82 e. Compared with the Bader charge of 1.81 e calculated for Fe in bulk goethite, these values do not indicate a significant transfer of electron density typical of an oxidation process.

As far as the projected DOS around the Fermi level is concerned, imposing an AFM 
TABLE IV: GGA-optimized bond lengths for $\left[\mathrm{Fe}\left(\mathrm{H}_{2} \mathrm{O}\right)_{6}\right]^{2+}$ bound to the goethite (110) and (021) surfaces, compared with the equivalent bonds in bulk goethite. Atoms are labelled as in fig. 4 .

\begin{tabular}{l|rr}
\hline \hline Bond & \multicolumn{2}{|c}{ Length $(\AA)$} \\
& Optimized Bulk \\
\hline$(110)$ surface: & & \\
$\mathrm{Fe}^{C}-\mathrm{O}^{1}$ & 2.003 & 1.971 \\
$\mathrm{Fe}^{C}-\mathrm{O}^{2}$ & 1.944 & 1.971 \\
$\mathrm{Fe}^{C}-\mathrm{Fe}^{A}$ & 3.729 & 3.427 \\
$\mathrm{Fe}^{C}-\mathrm{Fe}^{B}$ & 3.515 & 3.427 \\
\hline$(021)$ surface: & & \\
$\mathrm{Fe}^{C}-\mathrm{O}^{1}$ & 2.018 & 1.971 \\
$\mathrm{Fe}^{C}-\mathrm{O}^{2}$ & 2.029 & 2.113 \\
$\mathrm{Fe}^{C}-\mathrm{O}^{3}$ & 1.920 & 2.136 \\
$\mathrm{Fe}^{C}-\mathrm{Fe}^{A}$ & 3.179 & 3.365 \\
$\mathrm{Fe}^{C}-\mathrm{Fe}^{B}$ & 3.032 & 3.006 \\
\hline \hline
\end{tabular}

ordering between the surface and the added complex results in non-integer total spin of the system $\left(4.8 \mu_{B}\right)$, due to trapping of the HOMO of the complex and the previously vacant minority spin $\mathrm{Fe}(3 d)$-like surface states at the Fermi level. Reversing the spin direction of the complex (i.e. choosing the majority spin of the complex to be the same as that of the adjacent $\mathrm{Fe}$ ions in the surface) results in a total spin for the system of $4 \mu_{B}$. (The calculated total energy of this system is $0.09 \mathrm{eV}$ higher than in the previous case). This enables a weak interaction between the occupied minority spin $\mathrm{Fe}(3 d)$ orbital of the complex and the previously unoccupied $3 d$ orbitals of the neighboring Fe ions in the surface. With a smearing width of $0.01 \mathrm{eV}$, it is possible to resolve a HOMO (occupancy 0.90) and LUMO (occupancy 0.10 ), separated in energy by $0.025 \mathrm{eV}$. However, the weakness of the interaction combined with its half-metallic character lead us to believe that this is as an artifact arising from the unphysically small band gap in the electronic structure of goethite at the GGA level.

At the $\mathrm{GGA}+U$ level, irrespective of the majority spin direction of the complex, we 

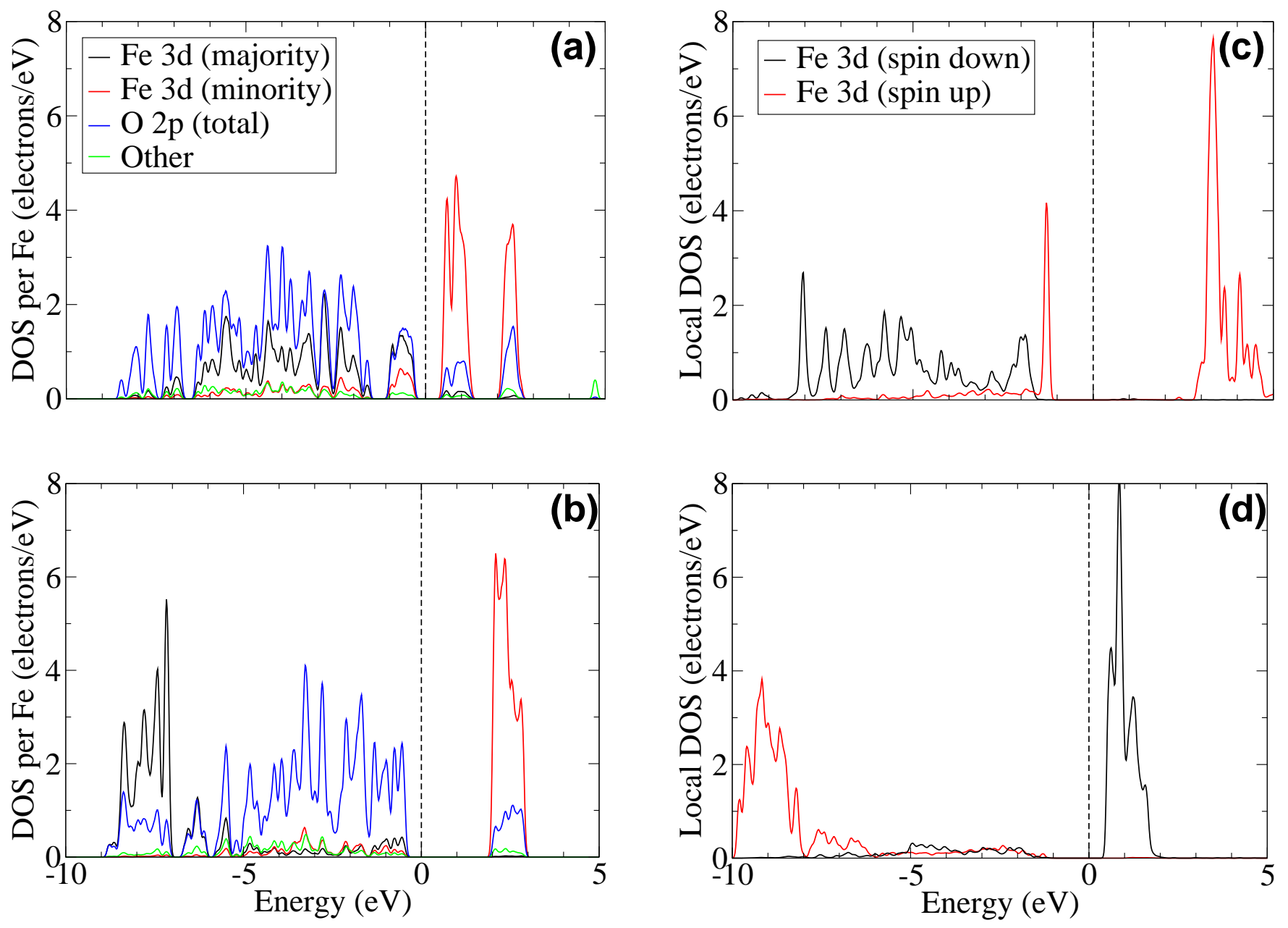

FIG. 5: (Color) Calculated electronic density of states projected onto local Fe( $3 d$ ) orbitals: (a) bulk goethite GGA, (b) bulk goethite $\mathrm{GGA}+U$, (c) complex on (110) GGA $+U$, (d) adjacent Fe for complex on (110) GGA $+U$.

obtain an integer total spin of $4 \mu_{B}$. In contrast to the GGA case, the Bader charge of the adsorbed Fe ion decreases slightly, from 1.67 to 1.61 e, while the charges on the surface Fe ions remain within 0.02 of the values for the bare surface. The projected density of states on the bound complex shows a single occupied $\mathrm{Fe}(3 d)$ minority spin orbital, characteristic of the Fe(II) oxidation state (Fig. 55). Meanwhile, the minority spin $d$-orbitals of the surface Fe ions remain safely above the Fermi level, as shown in fig. $5 \mathrm{~d}$. We therefore conclude that these ions remain in the Fe(III) oxidation state expected for bulk goethite. 

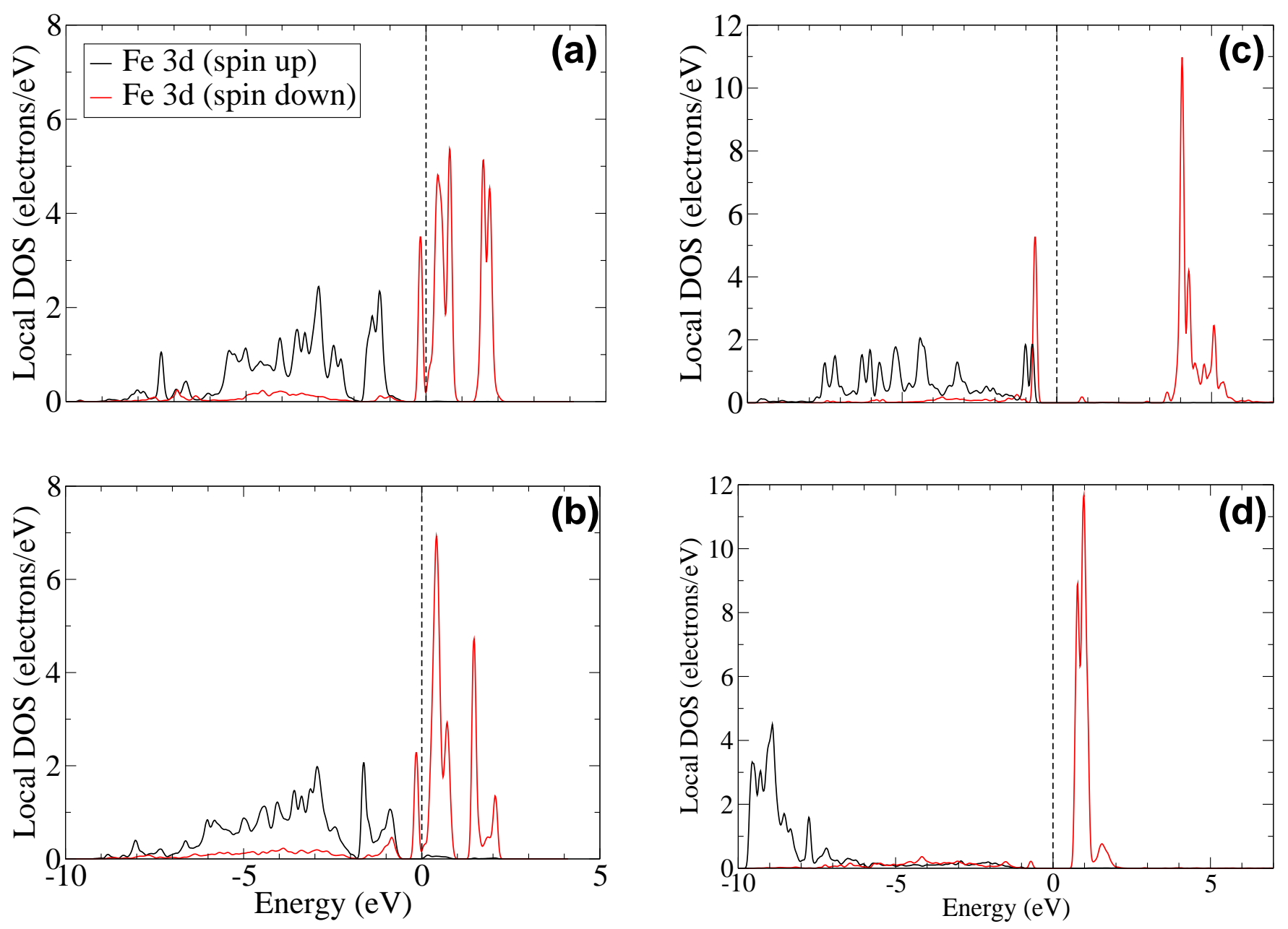

FIG. 6: (Color) Calculated electronic density of states projected onto local Fe(3d) orbitals: (a) complex on (021) GGA, (b) adjacent Fe for complex on (021) GGA, (c) complex on (021) GGA+U, (d) adjacent Fe for complex on (021) GGA+U.

\section{B. Double edge sharing on the (021) surface}

We now consider the double edge sharing site on the (021) surface, starting as before with the GGA results. On binding to the surface, we observe an increase of the Bader charge on the adsorbed complex, from 1.61 to $1.66 \mathrm{e}$, and a corresponding increase of the spin moment from 3.66 to $3.83 \mu_{B}$. More importantly, this is accompanied by a significant decrease in the Bader charge of the nearest surface Fe ion from 1.89 to 1.71 e. This value is 0.10 e lower than for an Fe ion in bulk goethite and is thus clear evidence for partial electron transfer from the complex to the oxide.

To investigate this issue further, we examine the density of electronic states projected 
(a)

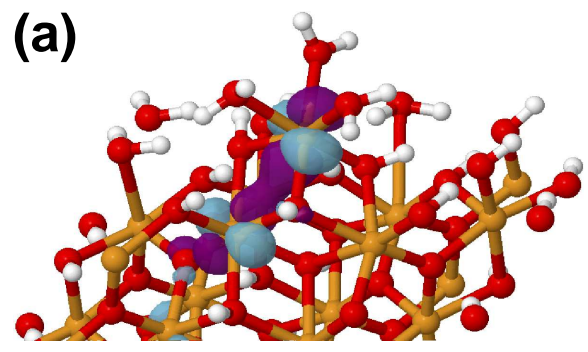

(b)

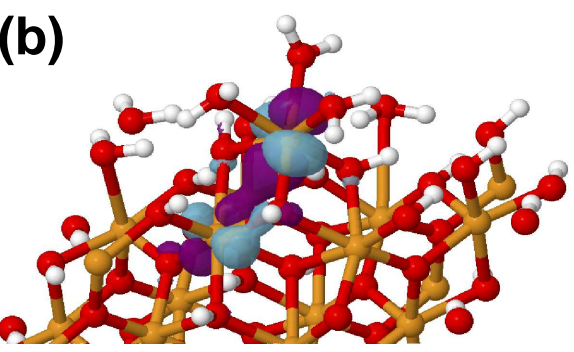

(c)

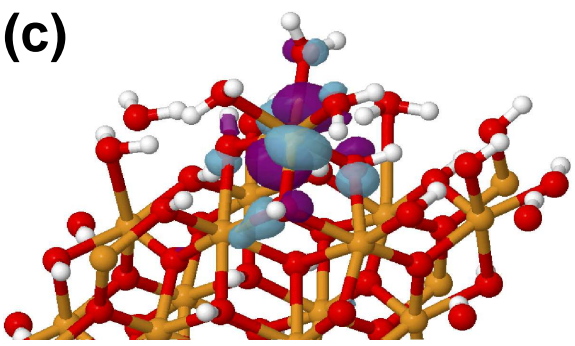

FIG. 7: (Color online) The HOMO for an Fe(II) hex-aqua complex bound to the goethite (021) surface, calculated by the GGA $+U$ method with (a) $U=0$, (b) $U=3.0 \mathrm{eV}$, (c) $U=5.2 \mathrm{eV}$.

onto atomic $3 d$-orbitals on the same two Fe ions, as shown in figs. 6a and 6b. On each ion, the projected DOS reveals an occupied majority spin $d$-shell and a mostly unoccupied set of minority spin levels, but with a single distinct minority spin peak $0.15 \mathrm{eV}$ below the Fermi level. We previously identified such a peak as a signature of the Fe(II) oxidation state, and its presence in the DOS of the surface Fe ion therefore indicates partial reduction of this $\mathrm{Fe}^{3+}$ ion after binding of the $\mathrm{Fe}^{2+}$ complex. This state, which represents the HOMO of the overall system, is localized over both ions, as depicted in fig. 77a. The integrated projected DOS amounts to 0.50 e on the adsorbed ion and 0.32 e on the adjacent surface ion (the remainder being attributable mainly to $\mathrm{O}(2 p)$ orbitals).

This behavior is not, however, reproduced at the GGA+U level with $U=5.2 \mathrm{eV}$. In this case, the Bader charge of the complex Fe ion is reduced from 1.67 to 1.56 e on binding to the surface. As before, the Bader charge of the adjacent surface Fe ion decreases from 1.99 in the bare surface to 1.91 with the adsorbed complex. However, unlike in the pure GGA case, this does not represent a significant reduction below the expected bulk value of 1.93. Consistently, the projected DOS on the surface ion (fig. 6 d) does not show an occupied minority spin $3 d$-orbital, and is seen to be very similar to the DOS previously obtained in the case of the (110) surface (fig. $5 \mathrm{~d}$ ). Instead, this feature is clearly evident on the adsorbed complex (fig. 6r), with an integrated DOS of 0.75 e. The HOMO state of the whole system at the GGA+U level is now visibly localized only on the adsorbed ion (fig. [7c), confirming that the added complex retains its Fe(II) oxidation state.

To test the sensitivity of these conclusions to the value of the Hubbard correction, we repeated the GGA $+U$ calculation with a lower value of $U=3.0 \mathrm{eV}$. With this value, we obtain a result intermediate between the GGA case the case with $U=5.2 \mathrm{eV}$. Namely, the 
HOMO (fig. 7b) shows significant interaction between minority spin $\mathrm{Fe}(3 d)$ orbitals on the adsorbed complex and the adjacent surface ion, although the orbital is weighted slightly more towards the complex than in the GGA ground state (the integrated DOS amounts to 0.65 and 0.25 e for the complex and surface ions, respectively) $\underline{\underline{53}}$

\section{Discussion}

In the two previous sections we looked for possible signatures of spontaneous oxidation of a $\left[\mathrm{Fe}\left(\mathrm{H}_{2} \mathrm{O}\right)_{6}\right]^{2+}$ complex after adsorption on either the (110) or (021) goethite surfaces. On the (110) surface, we find no evidence for significant electron transfer from the complex to the oxide at the GGA or GGA $+U$ levels. In this case, the Hubbard term appears to correct a presumed artefact arising from the severe underestimate of the band gap, which results in a semi-metallic behavior of the system.

On the other hand, the GGA results for the (021) surface show a much stronger overlap between minority spin $\mathrm{Fe}(3 d)$ orbitals on the complex and the neighboring surface Fe ion. As a result, one electron is delocalized between these two ions, which can be thought to be in a shared $\mathrm{Fe}(\mathrm{II})-\mathrm{Fe}(\mathrm{III})$ oxidation state. The effect of the $U$ correction in this case is to suppress such delocalization, to an extent which we found to depend strongly on the chosen value of $U$. In particular, using the value of $U=5.2 \mathrm{eV}$ optimized for bulk goethite, the delocalization is eliminated completely, but a value of $U=3.0 \mathrm{eV}$ still allows significant sharing of electron density between the complex and the surface. This illustrates the importance of choosing the "correct" value of $U$ for a given system in order to get an accurate description of the electronic structure.

The partial electron transfer observed for the (021) but not for the (110) surface seems to be consistent with studies of mixed valence minerals such as magnetite, which show that the electron sharing between neighboring ions arises from overlap of $\mathrm{Fe}(3 d)$ orbitals in octahedral edge-sharing environments. $\stackrel{54}{\underline{4}}$ In other words, the different behavior on the two surfaces may be due to the difference between the corner sharing (favored on the (110) surface) and the edge-sharing (favored on the (021) surface) adsorption sites. However, we have to note that the clear electron sharing observed at the GGA level is progressively reduced at the GGA $+U$ level as the Hubbard parameter $U$ increases. On the basis of our calculation, we are thus led to conclude that the Fe(II) hex-aqua complex is not spontaneously fully oxidized on binding 
to either of the two goethite surfaces.

In light of this conclusion, the experimental results recently reported by Wilson et al. $\underline{10}$ on the adsorption of Fe(II) complexes on Fe(III) oxyhydroxide surfaces are puzzling. As mentioned in the introduction, their Mössbauer spectroscopy study showed clear evidence for a transfer of electrons from hydrated $\mathrm{Fe}^{2+}$ ions to localized sites underneath the oxide surface. One possible explanation is that defects or vacancies in the crystal, not considered in our present study, might act as electron traps. Iron vacancies, for instance, are well known to be present in significant quantities even in well crystallized iron oxyhydroxides, where they play an important role in determining magnetic properties. ${ }^{55}$ In particular, a large concentration of defects was found to lower the Néel Temperature to $250 \mathrm{~K}$, which would imply a paramagnetic state of goethite at room temperature. $\underline{56}$ Further investigation is needed to determine whether defects could indeed enhance the ability of surrounding ions to accept electrons, and thus provide an explanation for the apparent discrepancy between the existing experimental finding and our simulations.

\section{OXIDATION BY MOLECULAR OXYGEN}

In the previous section we have addressed the possibility of spontaneous oxidation of an adsorbed $\mathrm{Fe}^{2+}$ hex-aqua complex on the goethite surface. Although some electron delocalization between complex and surface is observed in the case of adsorption on the (021) surface, no clear evidence for a net electron transfer process is found in the absence of defects. In this section, we investigate whether the surface, rather than drawing electrons away from the adsorbed Fe(II) complex, might instead increase its ability to lose electrons to an external oxidizing agent. In many cases where an iron oxyhydroxide phase grows from aqueous $\mathrm{Fe}(\mathrm{II})$, the net reaction taking place at the mineral surface involves oxidation by molecular oxygen:

$$
4 \mathrm{Fe}_{(\mathrm{aq})}^{2+}+\mathrm{O}_{2}+6 \mathrm{H}_{2} \mathrm{O} \rightarrow 4 \mathrm{FeOOH}_{(\mathrm{s})}+8 \mathrm{H}^{+}
$$

This equation does not represent a single step chemical process, but rather a complex multistage reaction, and a deep investigation of the full reaction mechanisms is outside the scope of the present work. We thus restrict ourselves to investigating a possible first step of the global reaction, namely the interaction of an oxygen molecule with a single $\mathrm{Fe}^{2+}$ complex, first isolated and then adsorbed onto the goethite (110) or (021) surfaces. 
The ground state of the oxygen molecule is a spin triplet, with one electron in each of two degenerate $\pi$-antibonding orbitals. Any additional electron donated from the complex to the molecule during the oxidation will be transferred to these orbitals. Increasing the occupation of the antibonding orbitals will weaken the $\mathrm{O}-\mathrm{O}$ bond, leading to an increase in the bond length and eventually, especially via interaction with solvent water molecules, to bond dissociation. Here, we are looking at how the interaction differs depending on the surface adsorption mode of the complexes, as this might give some insight into the catalytic role played by the mineral surface.

\section{A. Isolated Complexes}

Since Fe ions are almost universally observed to be six-fold coordinated in aqueous solution, we assume that an oxygen molecule will bind to an $\left[\mathrm{Fe}\left(\mathrm{H}_{2} \mathrm{O}\right)_{6}\right]^{2+}$ complex by substituting for one of the water ligands, forming the species $\left[\mathrm{FeO}_{2}\left(\mathrm{H}_{2} \mathrm{O}\right)_{5}\right]^{2+}$. The oxygen molecule may bind to the central Fe ion either in an end-on configuration via only one of the two oxygen atoms, or a side-on configuration, via both $\mathrm{O}$ atoms with (approximately) equal $\mathrm{Fe}-\mathrm{O}$ lengths. For both configurations, we assume that the majority spin direction of the Fe ion and the oxygen molecule prior to the interaction are aligned, giving a total spin of $6 / 2$, so that an electron can in principle be donated directly from the (minority spin) HOMO of the complex into one of the $\pi$-antibonding orbitals of the oxygen molecule.

The structure of both possible configurations in vacuo was optimized by means of GGA DFT calculations, using a cubic supercell of side length $12 \AA$ and a single k-point at the center of the Brillouin zone. In both cases, we observe an increase in the $\mathrm{O}-\mathrm{O}$ bond length from the reference value of $1.23 \AA$ (obtained for an isolated oxygen molecule), to $1.25 \AA$ and $1.29 \AA$ for the end-on and side-on configurations, respectively. The total energy of the relaxed system is lower by $0.14 \mathrm{eV}$ in the side-on configuration. The Bader charge on the central Fe atom is increased by 0.10 and 0.22 e upon binding in the end-on and side-on configuration, respectively, consistent with a small electron transfer to the oxygen molecule. Correspondingly, the net negative charge on the molecule increases by 0.14 and 0.30 e in the two respective cases. In both cases, the changes in the local spin moment of $\mathrm{Fe}$ are consistent with the changes in the charges and confirm that the observed electron transfer involves primarily minority spin electrons. 

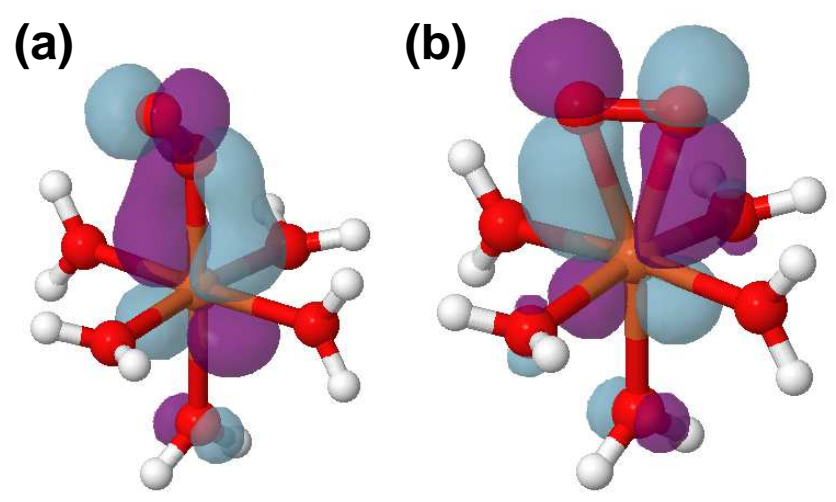

FIG. 8: (Color online) Highest occupied minority spin orbital of $\left[\mathrm{FeO}_{2}\left(\mathrm{H}_{2} \mathrm{O}\right)_{5}\right]^{2+}$, from GGA calculations, for end-on (a) and side-on (b) orientations of the oxygen molecule.

The minority spin orbitals responsible for bonding and the resulting electron transfer are shown in fig. 8, showing, as expected, strong overlap between an occupied $\mathrm{Fe}(3 d)$ and one of the unoccupied $\pi$-antibonding molecular orbitals on the oxygen molecule. The newly formed molecular orbital is of bonding character with respect to the $\mathrm{Fe}-\mathrm{O}$ bonds and antibonding with respect to the $\mathrm{O}-\mathrm{O}$ bond. The formation and occupation of this orbital rather than the original $\mathrm{Fe}(3 d)$-dominated HOMO of the hex-aqua complex therefore explains both the observed transfer of electron density from the Fe ion to the oxygen molecule and the resulting weakening of the $\mathrm{O}-\mathrm{O}$ bond. The stronger interaction in the side-on case may be attributed simply to greater overlap between the iron and oxygen orbitals in this configuration.

In contrast with the GGA results presented above, geometry optimization of the oxycomplexes at the GGA $+U$ level (using a value of $U=3.7 \mathrm{eV}$ determined self-consistently for an Fe(II) hex-aqua complex) causes the oxygen molecule in both configurations to dissociate spontaneously from the complex, with the five water ligands rearranging themselves to fill in the gap. We note that our simulations are performed in vacuo, and that the presence of further hydration shells may in principle influence the stability of the oxygenated complex. In order to obtain a reference point with which subsequent structures of complexes adsorbed to the surface will be compared, we performed a constrained geometry optimization of the side-on configuration, starting from the GGA-optimized structure and allowing only the two oxygen atoms of the oxygen molecule to move. In this case, the oxygen molecule remains bound, albeit very weakly, with an average $\mathrm{Fe}-\mathrm{O}$ distance of $2.34 \AA$ and an $\mathrm{O}-\mathrm{O}$ bond length of $1.26 \AA$. The oxygen molecule displays a net negative Bader charge of magnitude 0.12 e. 
The reduction in the interaction between the Fe ion and the oxygen molecule resulting from the Hubbard $U$ correction is most likely due to the lowering in energy of the minority spin $\mathrm{Fe}(3 d)$-dominated HOMO of the complex relative to the LUMO of the oxygen molecule. Indeed, increasing $U$ to $5.2 \mathrm{eV}$ (which is the self-consistent value for bulk goethite), the oxygen molecule remains electrostatically neutral and adopts a less symmetrical position with $\mathrm{Fe}-\mathrm{O}$ bond lengths of 2.59 and $2.75 \AA$ and an $\mathrm{O}-\mathrm{O}$ bond length of $1.24 \AA$.

\section{B. Adsorbed Complexes}

We now turn our attention to the interaction of an oxygen molecule with an $\mathrm{Fe}(\mathrm{II})$ aqua complex adsorbed on the (110) and (021) goethite surfaces in the two adsorbed geometries investigated in Section IV]. As for the isolated complexes, an oxygen molecule is substituted for one of the free water ligands. In light of the results of the previous section, the oxygen molecule is placed in a side-on orientation with respect to the central Fe ion, in order to maximize the resulting interaction.

Both systems were fully relaxed at the GGA level, leading to the structures shown in fig. 9. GGA $+U$ calculations were also carried out, using the same value of $U=5.2 \mathrm{eV}$ as previous calculations, starting from the optimized GGA structures and allowing only the $\mathrm{O}$ atoms of the bound $\mathrm{O}_{2}$ molecule to move according to the GGA $+U$ forces. In all cases, the $\mathrm{O}-\mathrm{O}$ bond length increases significantly relative to the reference gas-phase value of $1.23 \AA$. At the GGA level, the relaxed O-O distance is increased by $6.8 \%$ on either surface, compared with the increase of $4.6 \%$ obtained for the isolated complex. At the GGA $+U$ level the $\mathrm{O}-\mathrm{O}$ bond length increases by $3.6 \%$ on the (110) surface and by $4.3 \%$ on the (021) surface. Notably, for the isolated complex the increase was less than $1 \%$ at this value of $U$. Consistently with the weakening of the $\mathrm{O}-\mathrm{O}$ bond, the bound $\mathrm{O}_{2}$ molecule presents a Bader charge of -0.50 or -0.52 e at the GGA level for the (110) or (021) surfaces, respectively. At the GGA $+U$ level, the corresponding values are -0.26 and -0.34 e.

These results give good evidence that the transfer of electron density to the oxygen molecule and the resulting weakening of the $\mathrm{O}-\mathrm{O}$ bond are greatly enhanced for $\mathrm{Fe}(\mathrm{II})$ complexes bound to the goethite surface with respect to free solvated complexes. The effect appears to be slightly stronger on the (021) surface than on the (110) surface, especially in the $\mathrm{GGA}+U$ calculations, but the difference is small compared with the overall enhancement. 

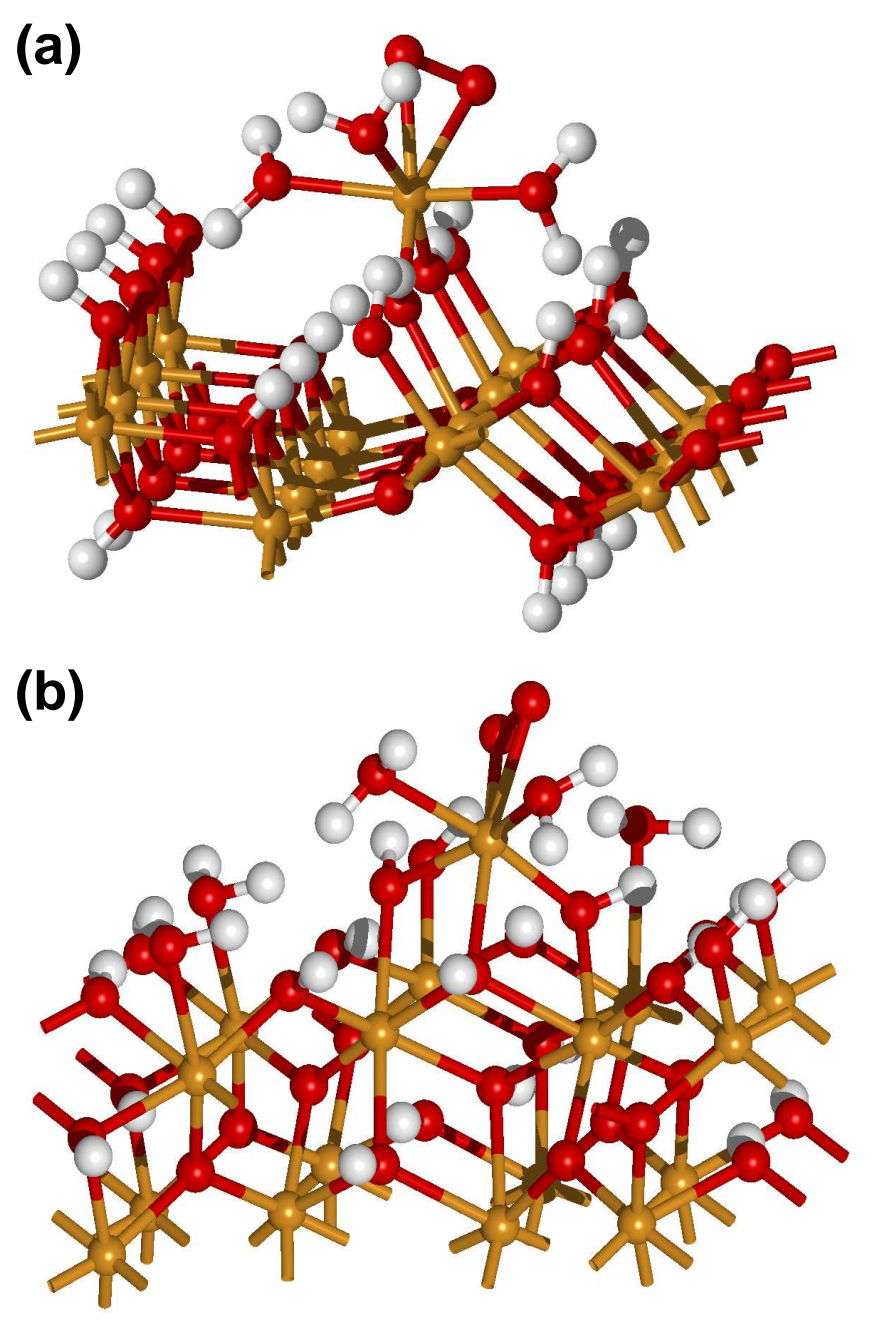

FIG. 9: (Color online) The GGA relaxed structure of $\left[\mathrm{FeO}_{2}\left(\mathrm{H}_{2} \mathrm{O}\right)_{5}\right]^{2+}$ bound to the goethite (110) surface in a double corner sharing configuration (a) and to the goethite (021) surface in a double edge sharing configuration (b).

In all cases the Bader analysis reveals an increase in the positive charge of the complex Fe ion resulting from the presence of the oxygen molecule. At the GGA $+U$ level, this is the first sign of partial oxidation of this ion in any of the systems studied. Interestingly, however, contrary to the charge donated to the oxygen molecule, the increase in the positive charge on the Fe atom is smaller for bound than for isolated complexes. Furthermore, the increases in the charge of the Fe ion are in all cases too small to account fully for the negative charge on the oxygen molecule.

To investigate this issue further, we look at the $\mathrm{Fe}(3 d)$ projected density of states for the oxy-complex adsorbed on either surface compared with an isolated $\mathrm{Fe}^{2+}$ hex-aqua com- 

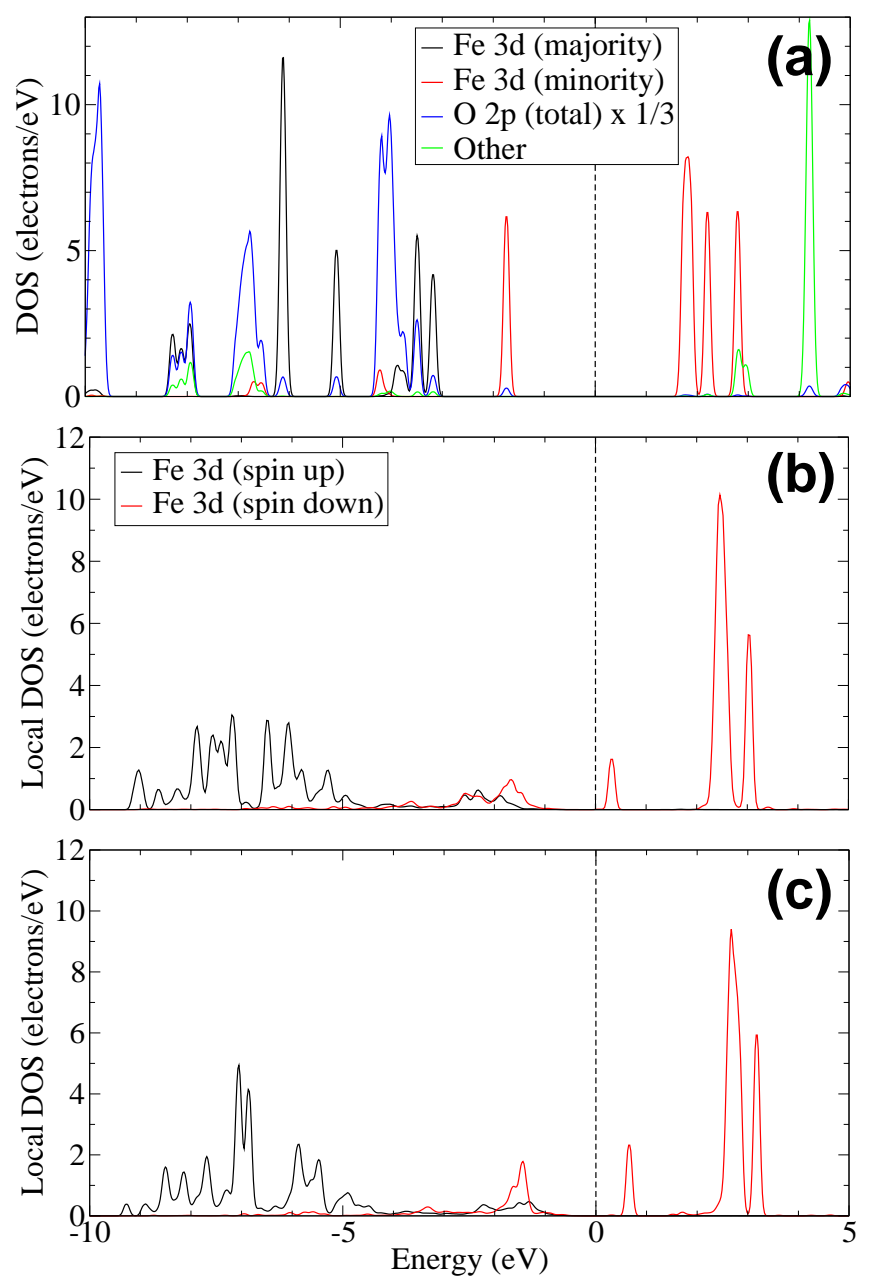

FIG. 10: (Color) Calculated electronic density of states $(\mathrm{GGA}+U)$ projected onto local $\mathrm{Fe}(3 d)$ orbitals: (a) $\left[\mathrm{Fe}\left(\mathrm{H}_{2} \mathrm{O}\right)_{6}\right]^{2+}$ complex, (b) oxy complex on (110) (c) oxy complex on (021).

plex. This is shown in Fig. 10 for the GGA $+U$ case (similar results were obtained at the simple GGA level). The DOS of the Fe(II) hex-aqua complex shows a single sharp peak in the minority spin density of states just below the Fermi level, representing the single occupied $3 d$ orbital that characterizes the Fe(II) oxidation state. The DOS of the oxygenated complexes show two smaller peaks either side of the Fermi level, corresponding to bonding and antibonding combinations with the $\pi$-antibonding orbital on the oxygen molecule. The occupation of only the bonding combination therefore represents a partial oxidation of the complex Fe ion, with the extra electron density donated to the oxygen molecule as expected.

It is also noteworthy that, in all cases, the surface Fe ion neighboring the oxy-complex remains in a state almost indistinguishable from bulk goethite. In the case of the (021) surface, this is significantly different from the behavior of this ion in the absence of a bound 
oxygen molecule (fig. 6b). As presented in Section 4, in that case the HOMO consisted of minority spin $\mathrm{Fe}(3 d)$ orbitals delocalized on the complex and on this ion, resulting in a partially shared Fe(II)-Fe(III) oxidation state between the two ions.

\section{Discussion}

When an oxygen molecule binds to an Fe(II) aqua complex, electron density is transfered into one of the $\mathrm{O}-\mathrm{O} \pi$-antibonding orbitals through overlap with the single occupied minority spin $\mathrm{Fe}(3 d)$ orbital. The oxygen molecule becomes negatively charged, and simultaneously the $\mathrm{O}-\mathrm{O}$ bond is weakened, rendering it vulnerable to hydrolysis. In our simulations, the donation of charge into the oxygen molecule is enhanced for complexes adsorbed onto either the (110) or the (021) surface of goethite, a result reproduced by both the GGA and the GGA $+U$ calculations. The effect is slightly stronger on the (021) surface, but the difference is only a small fraction of the overall effect. We thus conclude that the oxidation of Fe(II) by dioxygen may be catalyzed by adsorption onto a goethite surface, and that the strength of the catalytic effect is expected to vary little between the (110) and (021) surfaces.

The catalytic effect may thus depend negligibly on the details of the interaction between the complex and the surface, which are significantly different for the two cases, as presented in Section IV] We propose that the oxidation enhancement may result simply from the higher availability of electrons in the surface environment. Indeed, the fact that the negative charge on the oxygen molecule is only partially accounted for by the increase in the positive charge of the Fe(II) ion indicates that the electron density lost from the Fe(II) ion is compensated by donation of electrons from the remaining ligands. This donation process is energetically unfavorable in the presence of electronegative water ligands only, as for the isolated complex. However, ligands shared between the complex and the surface can gather electrons from the surrounding bulk oxide, and are therefore much better placed to act as electron donors. Indeed, on oxygenation of the (021) adsorbed complex, no Bader charge on the nearby atoms is changed by more than 0.02 e. This seems to confirm the idea that the additional electronic charge donated to the oxygen molecule is gathered from a larger area of Fe(III) oxide and not from any individual ion, similar to previous findings in the case of adsorbed $\mathrm{Sb}$ (III) $)^{57}$. 


\section{CONCLUSIONS}

We have performed a computational study within density functional theory of the stabilities of goethite surfaces and of their interaction with Fe(II) complexes in the context of FeOOH crystal growth from aqueous solutions. Background calculations on bulk goethite showed that structural properties computed at the GGA level are in good agreement with experiment. The size of the band gap and the nature of the states around the Fermi energy are not well described at this level of theory, but may be corrected by use of the GGA $+U$ method.

Ab initio thermodynamics calculations performed on the (110) and (021) surfaces of goethite predict full hydroxylation of both surfaces when in contact with liquid water. The free energy difference between the two surfaces is too small to account for the observed needle-like shape of goethite crystals, indicating that the crystal shape may be governed by kinetic factors rather than thermodynamic stability. Unequal growth rates of different surfaces could result either from different energy barriers for the adsorption of Fe complexes from solution or from different oxidation rates of adsorbed Fe(II) complexes.

Oxidation of adsorbed complexes by molecular oxygen on the (021) and (110) surfaces has been studied in Section V. In both cases we found that the underlying surface assists the transfer of electronic charge into the dioxygen molecule. With respect to isolated complexes in solution, bound complexes donate up to 0.34 electrons more into the oxygen molecule, rendering the $\mathrm{O}-\mathrm{O}$ bond increasingly susceptible to hydrolysis. The loss of electrons from the Fe ion to the oxygen molecule is compensated to a large degree by donation of electrons back onto the Fe ion through the surface ligands. This process may play a significant role in the observed autocatalytic growth of Fe(III) oxides from Fe(II) complexes in oxidizing solutions. However, the mechanism of catalysis appears to be independent of the details of the interaction between the complex and the surface, occurring to an almost equal extent on both the (021) and (110) surfaces.

The two surfaces show a slightly different behavior during adsorption of Fe(II) aquacomplexes. In this case, some partial sharing of electronic charge has been observed between the complex and the surface ions on the (021) surface but not on the (110) surface. However, this effect is limited, especially at the GGA+U level, and our calculations do not show spontaneous oxidation of the complex upon binding to the surface, in apparent conflict 
with experimental results. $\stackrel{10}{*}$ In light of this discrepancy, we propose that defects in the oxide structure, such as Fe vacancies, may play an important role in assisting electron transfer from adsorbing complexes by trapping electrons underneath the surface. Further investigation is needed to test the validity of this suggestion.

Finally, on the basis of the results presented in Section IIIB3 we propose that Fe ions from solution may adsorb more easily on the (021) surface than on the (110) surface. This is due to the presence of very weakly bound water molecules on the (021) surface, which may be displaced with virtually no energy barrier by binding complexes at room temperature. Therefore, given that neither spontaneous oxidation nor oxidation via molecular oxygen ap-

pear to proceed differently on the two surfaces studied, the evident anisotropy of goethite crystals may be explained simply by different adsorption rates of additional complexes from solution. This seems to be consistent with the fact that goethite crystals present approximately the same shape regardless of whether they are grown by precipitation from an Fe(III) solution or by oxidation of an Fe(II) solution. $\underline{\underline{9}} \underline{\underline{47}}$

\section{Acknowledgments}

This work has been supported by the EPSRC, UK, under grant No. GR/S61263/01. Part of the work has been carried out within the HPC-Europa Project No. RII3-CT-2003506079 , with the support of the European Community Research Infrastructure Action of the FP6. Computational resources were provided by the Cambridge HPC Service, UK, and by the Hochleistungsrechenzentrum Stuttgart and by the Zentrum fr Informationsdienste und Hochleistungsrechnen, Dresden, Germany. The work of L. C. C. is supported by the Deutschen Forschungsgemeinschaft within the Emmy-Noether Programme.

* Electronic address: bjr27@tcm.phy.cam.ac.uk

$\dagger$ Present address: Hybrid Materials Interfaces Group, Faculty of Production Engineering, University of Bremen, 28359 Bremen, Germany; Electronic address: colombi@hmi.uni-bremen.de

1 J. Majzlan, K.-D. Grevel, and A. Navrotsky, Am. Mineral. 88, 855 (2003).

2 C. van der Zee, D. R. Roberts, D. G. Rancourt, and C. P. Slomp, Geology 31, 993 (2003). 
3 M. Kosmulski, S. Durand-Viral, E. Maczka, and J. B. Rosenholm, J. Colloid Interface Sci. 271, $261(2004)$.

4 G. A. Waychunas, C. S. Kim, and J. F. Banfield, J. Nanopart. Res. 7, 409 (2005).

5 D. Mohan and C. U. Pittman Jr., J. Hazard. Mater. 142, 1 (2007).

6 R. M. Cornell and U. Schwertmann, The Iron Oxides: Structure, Properties, Reactions, Occurrences and Uses (Wiley-VCH, Weinheim, 2003).

7 N. Núñez, P. Tartaj, M. Morales, R. Pozas, M. Ocaña, and C. Serna, Chem. Mater. 15, 3558 (2003).

8 D. Thies-Weesie, J. de Hoog, M. Hernandez Mendiola, A. Petukhov, and G. Vroege, Chem. Mater. 19, 5538 (2007).

9 P. G. Weidler, S. J. Hug, T. P. Wetche, and T. Hiemstra, Geochim. Cosmochim. Acta 62, 3407 (1998).

10 A. G. B. Williams and M. M. Scherer, Environ. Sci. Technol. 38, 4782 (2004).

11 P. Hohenberg and W. Kohn, Phys. Rev. 136, B864 (1964).

12 W. Kohn and L. J. Sham, Phys. Rev. 140, A1133 (1965).

13 K. Terakura, T. Oguchi, A. R. Williams, and J. Kübler, Phys. Rev. B 30, 4734 (1984).

14 K. Terakura, A. R. Williams, T. Oguchi, and J. Kübler, Phys. Rev. Lett. 52, 1830 (1984).

15 S. L. Dudarev, G. A. Botton, S. Y. Savrasov, C. J. Humphreys, and A. P. Sutton, Phys. Rev. B 57, 1505 (1998).

16 V. I. Anisimov, J. Zaanen, and O. K. Andersen, Phys. Rev. B 44, 943 (1991).

17 V. I. Anisimov, I. V. Solovyev, M. A. Korotin, M. T. Czyżyk, and G. A. Sawatzky, Phys. Rev. B 48, 16929 (1993).

18 I. V. Solovyev, P. H. Dederichs, and V. I. Anisimov, Phys. Rev. B 50, 16861 (1994).

19 J. B. Forsyth, J. G. Hedley, and C. E. Johnson, J. Phys. C: Solid State Phys. 1, 179 (1968).

20 A. Szytula et al., Phys. Status Solidi B 26, 429 (1968).

21 D. M. Sherman, Geochim. Cosmochim. Acta 69, 3249 (2005).

22 K. L. Smith and R. A. Eggleton, Clay. Clay Miner. 31, 392 (1983).

23 U. Schwertmann, Clay Miner. 19, 9 (1984).

24 M. Amouric, A. Baronett, D. Nahon, and P. Didier, Clay. Clay Miner. 34, 45 (1986).

25 K. L. Smith, A. R. Milnes, and R. A. Eggleton, Clay. Clay Miner. 35, 418 (1987).

26 S. R. Randall, D. M. Sherman, K. V. Ragnarsdottir, and C. R. Collins, Geochim. Cosmochim. 
Acta 63, 2971 (1999).

27 S. J. Clark, M. D. Segall, C. J. Pickard, P. J. Hasnip, M. J. Probert, K. Refson, and M. C. Payne, Z. Kristallogr. 220, 567 (2005).

28 M. C. Payne, M. P. Teter, D. C. Allan, T. A. Arias, and J. D. Joannopoulos, Rev. Mod. Phys. 64, 1045 (1992).

29 J. P. Perdew, K. Burke, and M. Ernzerhof, Phys. Rev. Lett. 77, 3865 (1996).

30 D. Vanderbilt, Phys. Rev. B 41, 7892 (1990).

31 K. Laasonen, A. Pasquarello, R. Car, C. Lee, and D. Vanderbilt, Phys. Rev. B 47, 10142 (1993).

32 S. G. Louie, S. Froyen, and M. L. Cohen, Phys. Rev. B 26, 1738 (1982).

33 H. J. Monkhorst and J. D. Pack, Phys. Rev. B 13, 5188 (1976).

34 M. Cococcioni, Ph.D. thesis, International School for Advanced Studies (SISSA) (2002), http://www.sissa.it/cm/phd.php.

35 M. Cococcioni and S. de Gironcoli, Phys. Rev. B 71, 035105 (2005).

36 K. Reuter and M. Scheffler, Phys. Rev. B 65, 035406 (2001).

37 A. Ben-Naim and Y. Marcus, J. Chem. Phys. 81, 2016 (1984).

38 T. Nagai, H. Kagi, and T. Yamanaka, Am. Mineral. 88, 1423 (2003).

39 H. J. Kulik, M. Cococcioni, D. A. Scherlis, and N. Marzari, Phys. Rev. Lett. 97, 103001 (2006).

40 A. Rohrbach, J. Hafner, and G. Kresse, J. Phys.: Condens. Matter 15, 979 (2003).

41 S. Bocquet and S. J. Kennedy, J. Magn. Magn. Mater. 109, 260 (1992).

42 J. R. Rustad, A. R. Felmy, and B. P. Hay, Geochim. Cosmochim. Acta 60, 1563 (1996).

43 T. P. Trainor et al., Surf. Sci. 573, 204 (2004).

44 X.-G. Wang, W. Weiss, S. K. Shaikhutdinov, M. Ritter, M. Petersen, F. Wagner, R. Schlögl, and M. Scheffler, Phys. Rev. Lett. 81, 1038 (1998).

45 N. H. de Leeuw and T. G. Cooper, Geochim. Cosmochim. Acta 71, 1655 (2007).

46 S. J. Suresh and V. M. Naik, J. Chem. Phys. 113, 9727 (2000).

47 J.-F. Boily, J. Lützenkirchen, O. Balmès, J. Beattie, and S. Sjöberg, Colloid Surface Physicochem. Eng. Aspect 179, 11 (2001).

48 A. Manceau, K. L. Nagy, L. Spadini, and K. V. Ragnarsdottir, J. Colloid Interface Sci. 228, 306 (2000).

49 R. Bader, Atoms in Molecules: A Quantum Theory (Oxford University Press, 1990).

50 G. Henkelman, A. Arnaldsson, and H. Jónsson, Comput. Mater. Sci. 36, 354 (2006). 
51 E. Sanville, S. D. Kenny, R. Smith, and G. Henkelman, J. Comput. Chem. 28, 899 (2007).

52 R. S. Mulliken, J. Chem. Phys. 23, 1833 (1955).

53 Also in an additional calculation performed using an $U$ value of 5.2 for the Fe atoms of the goethite slab and an U value of 3.7 for the adsorbed Fe atom (corresponding to the self-consistent values of bulk goethite and isolated complexes, respectively) we found that the HOMO was predominantly localised on the adsorbed complex, with an integrated DOS of 0.77 e.

54 D. M. Sherman, Phys. Chem. Miner. 14, 355 (1987).

55 O. Özdemir and D. J. Dunlop, Geophys. Res. Lett. 23, 921 (1996).

56 G. H. Lee, S. H. Kim, B. J. Choi, S. H. Huh, Y. Chang, B. Kim, J. Park, S. J. Oh, J. Korean Phys. Soc. 45, 1019 (2004).

57 A.-K. Leuz, H. Mönch, and C. A. Johnson, Environ. Sci. Technol. 40, 7277 (2006). 
